\title{
LOCAL DECAY OF WAVES ON ASYMPTOTICALLY FLAT STATIONARY SPACE-TIMES
}

\author{
DANIEL TATARU
}

\begin{abstract}
In this article we study the pointwise decay properties of solutions to the wave equation on a class of stationary asymptotically flat backgrounds in three space dimensions. Under the assumption that uniform energy bounds and a weak form of local energy decay hold forward in time we establish a $t^{-3}$ local uniform decay rate for linear waves. This work was motivated by open problems concerning decay rates for linear waves on Schwarzschild and Kerr backgrounds, where such a decay rate has been conjectured by Price [39]. Our results apply to both of these cases.
\end{abstract}

\section{INTRODUCTION}

In this article we consider the question of pointwise decay for solutions to the wave equation on time independent, asymptotically flat backgrounds. Our interest in this problem comes from general relativity, more precisely the wave equation on Schwarzschild and Kerr backgrounds. There the conjectured decay, see Price [39, is $t^{-3}$, and numerous partial results have been obtained. Our main result applies to both the Schwarzschild and Kerr space-times, and proves a stronger form of Price's conjecture, where sharp bounds are provided in a full forward light cone. However, the result does not depend on the specific geometry of either Schwarzschild and Kerr space-times; instead, it applies to a large class of stationary asymptotically flat space-times.

We use $\left(x, t=x_{0}\right)$ for the coordinates in $\mathbb{R}^{3+1}$. We use Latin indices $i, j=1,2,3$ for spatial summation and Greek indices $\alpha, \beta=0,1,2,3$ for space-time summation. In $\mathbb{R}^{3}$ we also use polar coordinates $x=r \omega$ with $\omega \in \mathbb{S}^{2}$. By $\langle r\rangle$ we denote a smooth radial function which agrees with $r$ for large $r$ and satisfies $\langle r\rangle \geq 2$. We consider a partition of $\mathbb{R}^{3}$ into the dyadic sets $A_{k}=\left\{\langle r\rangle \approx 2^{k}\right\}$ for $k \geq 0$.

All operators we consider in this paper have time independent coefficients. To describe their spatial size and regularity we use the standard symbol classes $S\left(r^{k}\right)=S^{k}$ :

$$
a \in S\left(r^{k}\right) \Longleftrightarrow\left|\partial_{x}^{j} a(x)\right| \leq c_{j}\langle r\rangle^{k-j}, \quad j \geq 0
$$

By $S_{r a d}\left(r^{k}\right)$ we denote spherically symmetric functions in $S\left(r^{k}\right)$. We also introduce a slightly stronger class $l^{1} S\left(r^{k}\right)$ with some dyadic summability added,

$$
a \in l^{1} S\left(r^{k}\right) \Longleftrightarrow \sum_{m} 2^{m(j-k)}\left\|\partial_{x}^{j} a(x)\right\|_{L^{\infty}\left(A_{m}\right)} \leq c_{j}, \quad j \geq 0
$$

On occasion we allow for logarithmic modifications, and use the class of functions $S(\log r)$ :

$$
a \in S(\log r) \Longleftrightarrow|a| \lesssim \log \langle r\rangle, \quad\left|\partial_{x}^{j} a(x)\right| \leq c_{j}\langle r\rangle^{-j}, \quad j \geq 1
$$

The author was supported in part by the NSF grant DMS-0801261. 
We consider the pointwise decay problem for the forward wave equation

$$
\left(\square_{g}+V\right) u=0, \quad u(0)=u_{0}, \quad \partial_{t} u(0)=u_{1}
$$

associated to d'Alembertian $\square_{g}$ corresponding to to a Lorenzian metric $g$, and to a potential $V$. We work with potentials $V$ of the form

$$
V=V_{l r}+V_{s r}, \quad V_{l r} \in S_{r a d}\left(r^{-3}\right), \quad V_{s r} \in l^{1} S\left(r^{-3}\right)
$$

For the metric $g$ we consider two cases:

Case A: $g$ is a smooth Lorenzian metric in $\mathbb{R} \times \mathbb{R}^{3}$, with the following properties:

(i) $g$ is time independent, i.e. $\partial_{t}$ is a Killing vector field.

(ii) The level sets $t=$ const are space-like.

(iii) $g$ is asymptotically flat in the following sense:

$$
g=m+g_{s r}+g_{l r}
$$

where $m$ stands for the Minkowski metric, $g_{l r}$ is a long range spherically symmetric component, with $S_{\text {rad }}\left(r^{-1}\right)$ coefficients, of the form

$$
g_{l r}=g_{l r, t t}(r) d t^{2}+g_{l r, t r}(r) d t d r+g_{l r, r r}(r) d r^{2}+g_{l r, \omega \omega}(r) r^{2} d \omega^{2}
$$

and $g_{s r}$ is a short range component, with $l^{1} S\left(r^{-1}\right)$ coefficients, of the form

$$
g_{s r}=g_{s r, t t} d t^{2}+2 g_{s r, t i} d t d x_{i}+g_{s r, i j} d x_{i} d x_{j}
$$

We remark that these assumptions guarantee that $\partial_{t}$ is time-like near spatial infinity, but not necessarily in a compact set. This leads us to the second case we consider:

Case B: $g$ is a smooth Lorenzian metric $g$ in an exterior domain $\mathbb{R} \times \mathbb{R}^{3} \backslash B\left(0, R_{0}\right)$ which satisfies (i),(ii),(iii) above in its domain, and in addition

(iv) the lateral boundary $\mathbb{R} \times \partial B\left(0, R_{0}\right)$ is outgoing space-like.

This latter condition insures that the corresponding wave equation is well-posed forward in time. This assumption is satisfied in the case of the Schwarzschild and Kerr metrics in suitable advanced time coordinates, such as the ones used in [30, [49]. There the parameter $R_{0}$ is chosen so that $0<R_{0}<2 M$ in the case of the Schwarzschild metric, respectively $r^{-}<R_{0}<r^{+}$in the case of Kerr (see [19] for the definition of $r^{ \pm}$), so that the exterior of the $R_{0}$ ball contains a neighbourhood of the event horizon.

We consider the inhomogeneous forward Cauchy problem for the wave equation with initial data at time $t=0$,

$$
\left(\square_{g}+V\right) u=f, \quad u(0)=u_{0}, \quad \partial_{t} u(0)=u_{1}
$$

We denote the Cauchy data at time $t$ by $u[t]=\left(u(t), \partial_{t} u(t)\right)$. In the sequel $H^{k}=H^{k}\left(\mathbb{R}^{3}\right)$ is the usual Sobolev space, and by $\dot{H}^{k, 1}$ we denote the functions $u$ with $\nabla_{x} u \in H^{k}$. For the above evolution we begin with the following definition: 
Definition 1.1. We say that the evolution (1.3) is forward bounded if the following estimates hold:

$$
\|u[t]\|_{\dot{H}^{k, 1} \times H^{k}} \leq c_{k}\left(\|u[0]\|_{\dot{H}^{k, 1} \times H^{k}}+\|f\|_{L^{1} H^{k}}\right), \quad t \geq 0, \quad k \geq 0
$$

It suffices to have this property when $f=0$. Then the $f$ term can be added in by the Duhamel formula. The uniform forward bounds above are easy to establish provided that the Killing vector field $\partial_{t}$ is everywhere time-like. Otherwise, there is no general result, but various cases have been studied on a case by case basis.

In the case of the Schwarzschild space-time, following earlier work of Laba-Soffer [27, Blue-Soffer [3], 4, Twainy [52 and others, full uniform energy bounds were obtained by Dafermos-Rodnianski [16] in the exterior region. For a shorter alternate proof, as well as a discussion concerning the extension of the energy bounds to a neighbourhood of the event horizon we refer the reader to the article [30] of the author and collaborators.

As far as the Kerr space-time is concerned, partial results were first obtained by Finster-Smoller [18, and Finster-Kamran-Smoller-Yau [17. Full uniform energy bounds were proved in the the case of the Kerr space-time with small angular momentum in Dafermos-Rodnianski [14; their result applies in effect to a larger class of small perturbations of the Schwarzschild space-time. An alternate proof of the Kerr result, based on local energy decay estimates, was given shortly afterward in Tataru-Tohaneanu [49.

A stronger property of the wave flow is local energy decay. We introduce the local energy norm $L E$ by

$$
\|u\|_{L E}=\sup _{k}\left\|\langle r\rangle^{-\frac{1}{2}} u\right\|_{L^{2}\left(\mathbb{R} \times A_{k}\right)}
$$

its $H^{1}$ counterpart,

$$
\|u\|_{L E^{1}}=\|\nabla u\|_{L E}+\left\|\langle r\rangle^{-1} u\right\|_{L E}
$$

as well as the dual norm

$$
\|f\|_{L E^{*}}=\sum_{k}\left\|\langle r\rangle^{\frac{1}{2}} f\right\|_{L^{2}\left(\mathbb{R} \times A_{k}\right)}
$$

These definitions are specific to the three dimensional problems. Some appropriate modifications are needed in other dimensions, see for instance [34.

We also define similar norms for higher regularity,

$$
\|u\|_{L E^{1, k}}=\sum_{|\alpha| \leq k}\left\|\partial^{\alpha} u\right\|_{L E^{1}}
$$

respectively

$$
\|f\|_{L E^{*, k}}=\sum_{|\alpha| \leq k}\left\|\partial^{\alpha} f\right\|_{L E^{*}}
$$

The dual spatial norms $\mathcal{L E}$ and $\mathcal{L E}^{*}$ are the spatial counterparts of the space-time $L E$ and $L E^{*}$ norms,

$$
\|v\|_{\mathcal{L E}}=\sup _{k}\left\|\langle r\rangle^{-\frac{1}{2}} v\right\|_{L^{2}\left(A_{k}\right)} \quad\|f\|_{\mathcal{L E}^{*}}=\sum_{k}\left\|\langle r\rangle^{\frac{1}{2}} f\right\|_{L^{2}\left(A_{k}\right)}
$$


Their higher regularity versions are defined as

$$
\|v\|_{\mathcal{L} \mathcal{E}^{m}}=\sum_{j=0}^{m} \sup _{k}\left\|\langle r\rangle^{-\frac{1}{2}} \partial_{x}^{j} v\right\|_{L^{2}\left(A_{k}\right)} \quad\|f\|_{\mathcal{L E}^{*}, m}=\sum_{j=0}^{m} \sum_{k}\left\|\langle r\rangle^{\frac{1}{2}} \partial_{x}^{j} f\right\|_{L^{2}\left(A_{k}\right)}
$$

In Case $\mathrm{A}$ above this leads to the following

Definition 1.2. a) We say that the evolution (1.3) has the local energy decay property if the following estimate holds:

$$
\|u\|_{L E^{1, k}} \leq c_{k}\left(\|u[0]\|_{H^{1, k} \times H^{k}}+\|f\|_{L E^{*, k}}\right), \quad k \geq 0
$$

in $\mathbb{R} \times \mathbb{R}^{3}$.

The first local energy decay estimates for the wave equation were proved in the work of Morawetz 35, 36, 37. There is by now an extensive literature devoted to this topic and its applications; without being exhaustive we mention Strauss [43, Keel-SmithSogge [22, [23], Burq-Planchon-Stalker-Tahvildar-Zadeh [6], Metcalfe-Sogge [31, 32].

The sharp form of the estimates as well as the notations above are from MetcalfeTataru 34; this paper also contains a proof of the local energy decay estimates for small (time dependent) long range perturbations of the Minkowski space-time, and further references. See also the related paper Metcalfe-Tataru [33, as well as Alinhac's article [1].

There is a related family of local energy estimates for the Schrödinger equation. As a starting point we refer the reader to [48] and references therein.

Work in progress of Metcalfe-Tataru suggests that in the nontrapping stationary case the local energy decay (1.9) is closely tied to a non-resonant spectral behavior at frequency 0 (see 29] for our similar results for Schrödinger evolutions).

In Case B an estimate such as (1.9) is unlikely to hold due to the existence of trapped rays, i.e. null geodesics confined to a compact spatial region. However a weaker form of the local energy decay may still hold if the trapped null geodesic are hyperbolic. This is the case for both the Schwarzschild metric and for the Kerr metric with angular momentum $|a|<M$. To state such bounds we introduce a weaker version of the local energy decay norm

$$
\|u\|_{L E_{w}^{1}}=\|(1-\chi) \nabla u\|_{L E}+\left\|\langle r\rangle^{-1} u\right\|_{L E}
$$

for some spatial cutoff function $\chi$ which is smooth and compactly supported. Heuristically, $\chi$ is chosen so that it equals 1 in a neighbourhood of the trapped set. We define as well a dual type norm

$$
\|f\|_{L E_{w}^{*}}=\|\chi f\|_{L^{2} H^{1}}+\sum_{k}\left\|\langle r\rangle^{\frac{1}{2}} f\right\|_{L^{2}\left(A_{k}\right)}
$$

As before we define the higher norms $L E_{w}^{1, k}$ respectively $L E_{w}^{*, k}$.

Definition 1.3. We say that the evolution (1.3) has the weak local energy decay property if the following estimate holds:

$$
\|u\|_{L E_{w}^{1, k}} \leq c_{k}\left(\|u[0]\|_{\dot{H}^{1, k} \times H^{k}}+\|f\|_{L E_{w}^{*, k}}\right), \quad k \geq 0
$$

in either $\mathbb{R} \times \mathbb{R}^{3}$ or in the exterior domain case. 
Two examples where weak local energy decay is known to hold are the Schwarzschild space-time, and the Kerr space-time with small angular momentum $|a| \ll M$.

The estimates in the Schwarzschild case are the end result of a series of papers by LabaSoffer [27, Blue-Soffer [3], 4], 38, Blue-Sterbenz [5] Dafermos-Rodnianski [16], 13] and Marzuola-Metcalfe-Tataru-Tohaneanu [30. In particular we note the contribution of [16] to the understanding of the red shift effect near the event horizon, as well as that of 38, 30] concerning improved bounds near the photon sphere (i.e. the trapped set). The sharpest form of the estimates is obtained in [30; in particular the bounds there fit into the framework of Definition 1.3 .

In the case of the Kerr space-time with small angular momentum $|a| \ll M$ the local energy estimates were first proved in Tataru-Tohaneanu 49, in a form which is compatible with Definition 1.3. A more detailed presentation as well as stronger bounds near the trapped set are contained in Tohaneanu's Ph.D Thesis [50] as well as in the follow-up paper [51]. For related subsequent work we also refer the reader to Dafermos-Rodnianski [12] and Andersson-Blue [2].

The high frequency analysis of the dynamics near the hyperbolic trapped orbits has a life of its own, but not much to do with the present article. For more information we refer the reader to work of de Verdière-Parisse [11, Christianson [9], NonnenmacherZworski [42] and references therein.

We remark that the local energy decay property is stronger than the mere boundedness of the evolution. This is not as clear in the case of the weak local energy decay; however, one case which is well understood is when the Killing vector field $\partial_{t}$ is time-like within the support of the cutoff function $\chi$ in the definition of $L E_{w}^{1}$.

In this article we consider the pointwise decay of solutions to the wave equation (1.3). If $g$ is the Minkowski space-time then, by Huygens principle, if the initial data has compact support then $u$ will vanish in a compact set after some time. If instead we add a stationary potential which has exponential decay at infinity and no eigenvalues or zero resonances, then one obtains exponential decay of solutions inside any compact set. For polynomially decaying stationary perturbations of the Minkowski space-time the question of the precise decay rates is still largely open. In principle, one expects that these decay rates will be determined by three factors: (i) the spectral behavior near time frequency 0 (e.g. eigenvalues, resonances), (ii) the trapping properties of the metric and (iii) the behavior of the metric at spatial infinity.

This work was inspired by an idea in Tataru [48] and Metcalfe-Tataru 34, which is roughly that the local energy estimates contain all the important local and low frequency information concerning the flow, and that only leaves the analysis near spatial infinity to be understood. Precisely, in [48] and 34] it is proved that local energy decay implies Strichartz estimates in the asymptotically flat setting, first for the Schrödinger equation and then for the wave equation. The same idea was exploited in [30] and [51] to prove Strichartz estimates for the wave equation on the Schwarzschild and then on the Kerr space-time. 
The result we obtain in this article shows that the above philosophy applies as well to the pointwise local decay. Precisely, we prove that if weak local energy decay estimates hold, then the pointwise decay rate of the solutions to the wave equation is only determined by the behavior of the perturbation at spatial infinity.

To define the initial data spaces we use several vector fields: the generators of spatial translations $T=\left\{\partial_{1}, \partial_{2}, \partial_{3}\right\}$, the generators of rotations $\Omega=\left\{x_{i} \partial_{j}-x_{j} \partial_{i}\right\}$ and the spatial scaling $S_{r}=r \partial_{r}$. Then we define the weighted Sobolev spaces $Z^{m, k}$ with norms

$$
\|f\|_{Z^{m, n}}=\sup _{i+j+k \leq m}\left\|\langle r\rangle^{n} T^{i} \Omega^{j} S_{r}^{k} f\right\|_{\mathcal{L} \mathcal{E}^{*}}
$$

Now we are ready to state the main result of this article:

Theorem 1.4. Let $m$ be a large enough integer. Let $g$ be a metric which satisfies the conditions (i), (ii), (iii) in $\mathbb{R} \times \mathbb{R}^{3}$, or (i), (ii), (iii), (iv) in $\mathbb{R} \times \mathbb{R}^{3} \backslash B\left(0, R_{0}\right)$. Let $V$ be a potential as in (1.2). Assume that the evolution (1.3) is forward bounded and satisfies the weak local energy decay estimates (1.10). Then in normalized coordinates the solution $u$ to the homogeneous problem (1.1) satisfies the bounds

$$
|u(t, x)| \lesssim \frac{1}{\langle t+|x|\rangle\langle t-|x|\rangle^{2}}\left(\left\|u_{0}\right\|_{Z^{m+1,1}}+\left\|u_{1}\right\|_{Z^{m, 2}}\right)
$$

respectively

$$
\left|\partial_{t} u(t, x)\right| \lesssim \frac{1}{\langle t+|x|\rangle\langle t-|x|\rangle^{3}}\left(\left\|u_{0}\right\|_{Z^{m+1,1}}+\left\|u_{1}\right\|_{Z^{m, 2}}\right)
$$

The normalized coordinates referred to in the theorem are introduced in the next section via a spherically symmetric change of coordinates. These are needed in order to insure that the outgoing light cones are close to Minkowski cones.

The required decay of the initial data at infinity is roughly a summable $r^{-3}$ for the position $u_{0}$, respectively a summable $r^{-4}$ for the velocity $u_{1}$. These are sharp. By contrast, we made no effort to optimize the value of $m$.

In view of the local energy decay estimates in Marzuola-Metcalfe-Tataru-Tohaneanu 30, respectively Tataru-Tohaneanu [4], the hypothesis of the theorem is valid in the case of the Schwarzschild space time, and also the Kerr space-time with small angular momentum, with $V=0$. Thus we obtain:

Corollary 1.5 (Price's Law). The decay estimates (1.12), respectively (1.13) hold for the Schwarzschild space-time, as well as for the Kerr space-time with small angular momentum, with respect to coordinates which coincide with the Regge-Wheeler coordinates near spatial infinity and with the Eddington-Finkelstein coordinates near the event horizon.

This problem has had a long history. Dispersive $L^{1} \rightarrow L^{\infty}$ estimates providing $t^{-1}$ decay of $3+1$ dimensional waves in the Minkowski setting have been known for a long time. There is an extensive literature concerning dispersive estimates for $\square+V$; this is by now a well understood problem, for which we refer the reader to the survey paper of Schlag [1].

The need for weighted decay inside the cone arose in John's proof [20] of the Strauss conjecture in $3+1$ dimensions. Decay bounds for $\square+V$, similar to those in the theorem, 
were obtained by Strauss-Tsutaya 44] and Szpak [46, 45], see also Szpak-Bizoń-ChmajRostworowski 47 .

A more robust way of proving pointwise estimates via $L^{2}$ bounds and Sobolev inequalities was introduced in the work of Klainerman, who developed the so-called vector field method, see for instance 24]. This idea turned out to have a myriad of applications, and played a key role in the Christodoulou-Klainerman [10] proof of the asymptotic stability of the Minkowski space time for the vacuum Einstein equations.

In the context of the Schwarzschild space-time, Price conjectured the $t^{-3}$ decay rate for linear waves. More precise heuristic computations were carried out later by ChingLeung-Suen-Young [7, 8]. Following work of Wald [53, the first rigorous proof of the boundedness of the solutions to the wave equation was given in Kay-Wald 21].

Uniform pointwise $t^{-1}$ decay estimates were obtained by Blue-Sterbenz [38] and also Dafermos-Rodnianski [16]; the bounds in the latter paper are stronger in that they extend uniformly up to the event horizon. Very recently, a local $t^{-\frac{3}{2}}$ decay result was obtained by Luk [28]. These results are obtained using multiplier techniques, related to Klainerman's vector field method; in particular the conformal multiplier plays a key role.

Another venue which was explored was to use the spherical symmetry in order to produce an expansion into spherical modes, and to study the corresponding ode. This was pursued by Kronthaler [26, 25, who in the latter article was able to establish the sharp Price's Law in the spherically symmetric case. A related analysis was carried out later by Donninger-Schlag-Soffer [40] for all the spherical modes; they establish a $t^{-2}$ local decay for each spherical mode.

Switching to Kerr, the first decay results there were obtained by Finster-KamranSmoller-Yau [17. Later Dafermos-Rodnianski [12] were able to extend their Schwarzschild results to Kerr, obtaining almost a $t^{-1}$ decay.

Finally, we mention the related work of Dafermos-Rodnianski [15, where Price's Law is established in a nonlinear but spherically symmetric context.

We conclude the introduction with a brief overview of the paper. In the next section we discuss the normalization of the coordinate system, which is done in order to insure that the outgoing null cones are close to Minkowski cones. In the following section we use the uniform forward energy bounds in order to define resolvent operators $R_{\tau}$ associated to the evolution (1.1) in the lower half-space $\{\Im \tau<0\}$, and to establish straightforward $L^{2}$ and $H^{m}$ bounds for the resolvent. These bounds degenerate as $\Im \tau$ approaches 0 , so they provide no information about the resolvent on the real axis.

In Section 4 we transfer the local energy decay estimates to the resolvent $R_{\tau}$. These bounds have the key advantage that they are uniform as $\tau$ approaches the real axis.

Using a version of the vector field method, in Proposition 5.10 we commute the resolvent with the rotations $\Omega$ and scaling $S$ to obtain a larger class of resolvent bounds. These bounds allow us in Proposition 5.11 to prove that we can extend the resolvent $R_{\tau}$ to the real axis continuously in a weaker topology as well as show that it inherits all the estimates previously proved in the lower half space. We also establish the key outgoing radiation condition (5.34) for $R_{\tau}$ for real $\tau$. 
The limit $R_{0}$ of the resolvent $R_{\tau}$ as $\tau \rightarrow 0$ is considered in Propositions 6.13, 6.14 this allows us to define $R_{0}$, and establish some key properties for it. The precise description of $R_{0}$ is used in Proposition 6.15 to produce an expansion up to second order for the resolvent $R_{\tau}$ near $\tau=0$.

In Section 7 we switch from $L^{2}$ to $L^{\infty}$ bounds in the resolvent estimates for real $\tau$, in a way which is reminiscent of the Klainerman-Sobolev embeddings. Exploiting the outgoing radiation condition (5.34), we also obtain improved decay estimates at spatial infinity for $\left(\partial_{r}-i \tau\right)^{m} R_{\tau}$.

In the last section we conclude the argument. Assuming for simplicity that $u_{0}=0$, the time Fourier transform of the solution $u$ to the forward wave equation (1.1) is represented as $\hat{u}=R_{\tau} u_{1}$. Thus in order to invert the Fourier transform and obtain pointwise estimates for $u$ we need to control the size and regularity with respect to $\tau$ of $R_{\tau} u_{1}$. This is relatively easy for large $\tau$ because we allow the initial data to have high Sobolev regularity in Theorem 1.4. The delicate issue is the resolvent behavior at $\tau=0$. In order to obtain a $t^{-3}$ decay for $u$ at infinity, we use the second order expansion for $R_{\tau}$ near $\tau=0$ given by Proposition 6.15. In particular we are able to identify the leading contribution of the long range part of the wave operator and of the potential.

\section{The COORDINATE NORMALIZATION}

Here we carry out a preliminary step which brings the metric to a canonical form near spatial infinity and removes certain logarithmic type corrections in the resolvent bounds later on. This is achieved in two steps, a spherically symmetric coordinate change followed by a conjugation with respect to a scalar weight. This transformation is roughly equivalent to using Regge-Wheeler coordinates in Schwarzschild/Kerr near spatial infinity.

Our final goal is to reduce the problem to the case when the operator $\square_{g}+V$ in (1.1) is replaced by an $L^{2}$ selfadjoint operator $P$ which has the form

$$
P=-\partial_{t}^{2}+\Delta_{x}+P_{l r}+P_{s r}
$$

where the long range spherically symmetric part has the form

$$
P_{l r}=g_{l r}^{\omega}(r) \Delta_{\omega}+V_{l r}(r), \quad g_{l r}^{\omega}, V_{l r} \in S_{r a d}\left(r^{-3}\right)
$$

and the selfadjoint short range part $P_{s r}$ has the form

$$
P_{s r}=\partial_{\alpha} g_{s r}^{\alpha \beta} \partial_{\beta}+V_{s r}, \quad g_{s r}^{\alpha \beta} \in l^{1} S\left(r^{-1}\right), \quad V_{s r} \in l^{1} S\left(r^{-3}\right)
$$

with $g_{s r}^{00}=0$.

2.1. Normalized coordinates. We first remark that the condition (iii) translates into a similar representation for the dual coefficients $g^{\alpha \beta}$,

$$
g^{\alpha \beta}=m^{\alpha \beta}+g_{l r}^{\alpha \beta}+g_{s r}^{\alpha \beta}
$$

We can simplify these coefficients via a radial change of coordinates as follows:

Lemma 2.6. There exists a spherically symmetric change of coordinates so that the long range component $g_{l r}^{\alpha \beta}$ of $g^{\alpha \beta}$ has the additional properties

$$
g_{l r}^{t t}=-g_{l r}^{r r}, \quad g_{l r}^{r t}=0
$$


for large $r$.

We will refer to the coordinates given by this lemma as normalized coordinates. These are the coordinates used in Theorem 1.4. In Schwarzschild or Kerr space-times these coordinates are precisely the Regge-Wheeler coordinates for large $r$. In a bounded spatial region, any choice of coordinates which is nonsingular on the event horizon will suffice.

Proof. To achieve the relation $g_{l r}^{r t}=0$ we keep $r$ unchanged and reset $t$ by setting

$$
t:=t+a(r)
$$

where $a$ is determined by

$$
a^{\prime}(r)=-\frac{g_{l r}^{r t}}{1+g_{l r}^{r r}}
$$

Then we have $a^{\prime} \in S_{\text {rad }}\left(r^{-1}\right)$ and $a \in S_{\text {rad }}(\ln r)$, therefore the property (iii) is left unchanged. We remark that this can only be done provided that $1+g_{l r}^{r r}>0$. This is clearly true for large $r$, and can be arranged for small $r$ by modifying the short range part $P_{s r}$.

Once $g_{l r}^{r t}=0$, we insure that $g_{l r}^{r r}=g_{l r}^{t t}$ with a radial change of coordinates

$$
d r^{*}=\left(\frac{1-g_{l r}^{t t}}{1+g_{l r}^{r r}}\right)^{\frac{1}{2}} d r
$$

This gives $r^{*}-r \in S_{\text {rad }}(\ln r)$ and $d r^{*} / d r \in 1+S_{\text {rad }}\left(r^{-1}\right)$. Thus while both transformations above leave condition (iii) unchanged, both the functions $a(r)$ and $r^{*}(r)-r$ may have a logarithmic component as $r \rightarrow \infty$. The proof is concluded.

2.2. Left and right multiplication by scalar functions. Consider a metric $g$ as in (2.17). The corresponding d'Alembertian has the form

$$
\square_{g}=\frac{1}{\sqrt{g}} \partial_{\alpha} \sqrt{g} g^{\alpha \beta} \partial_{\beta}
$$

where it is easy to see that $g \in 1+S_{\text {rad }}\left(r^{-1}\right)+l^{1} S\left(r^{-1}\right)$. This is turned into an $L^{2}$ selfadjoint operator via the conjugation

$$
\square_{g} \rightarrow g^{\frac{1}{4}} \square_{g} g^{-\frac{1}{4}}
$$

To further insure that the coefficient $g_{l r}^{t t}$ of $\partial_{t}^{2}$ is set to -1 we multiply the above conjugated operator by $\left(-g^{t t}\right)^{-1} \in 1+l^{1} S\left(r^{-1}\right)$; in order to preserve the $L^{2}$ selfadjointness, half of it will go on the left and half will go on the right.

Thus, our final replacement for $\square_{g}+V$ is the operator

$$
P=g^{\frac{1}{4}}\left(-g^{t t}\right)^{-\frac{1}{2}}\left(\square_{g}+V\right)\left(-g^{t t}\right)^{-\frac{1}{2}} g^{-\frac{1}{4}}
$$

Commuting, $P$ can be written in divergence form, and it will have the required form given by (2.14), (2.15) and (2.16).

Also it is easy to see that such a transformation leaves unchanged the three properties of the forward evolution (1.3): boundedness, local energy decay and pointwise decay. 


\section{The RESOlVENT AND ENERGY ESTIMATES}

Consider the solution $u$ to the forward homogeneous problem

$$
P u=0, \quad u(0)=0, u_{t}(0)=g \in L^{2},
$$

extended by 0 to negative times.

By the uniform energy bounds its time Fourier transform $\hat{u}(\tau)$ is a $\dot{H}^{1}$ valued distribution in $\mathbb{R}$, and admits a holomorphic extension into the lower half plane. We define the resolvent operator $R_{\tau}$ by

$$
R_{\tau} g=\hat{u}(\tau), \quad \Im \tau<0
$$

The uniform energy bounds (1.9) for $P$ translate into an $L^{2}$ bound for $R_{\tau}$,

$$
\left\|R_{\tau} g\right\|_{\dot{H}^{1}}+\tau\left\|R_{\tau} g\right\|_{L^{2}} \lesssim \frac{1}{|\Im \tau|}\|g\|_{L^{2}}, \quad \Im \tau<0,
$$

as well as similar bounds for higher Sobolev norms,

$$
\left\|R_{\tau} g\right\|_{\dot{H}^{k, 1}}+\tau\left\|R_{\tau} g\right\|_{H^{k}} \lesssim \frac{1}{|\Im \tau|}\|g\|_{H^{k}}, \quad \Im \tau<0 .
$$

In order to write an equation for the resolvent we express the operator $P$ in the form

$$
P=-\partial_{t}^{2}+P^{1} \partial_{t}+P^{2}
$$

where, in view of (2.14), 2.15) and (2.16), $P^{1}$ is a short range spatial skew-adjoint operator and $P_{2}$ a second order spatial operator of the form

$$
P^{2}=\Delta+P_{l r}+P_{s r}^{2}
$$

with both long and short range components.

Since $u$ is extended by 0 to negative times, it solves the distributional equation

$$
P u=g \delta_{t=0}
$$

Taking a Fourier transform in this relation, it follows that $\hat{u}(\tau)$ solves the equation

$$
P_{\tau} \hat{u}(\tau)=g, \quad P_{\tau}=\tau^{2}-i \tau P^{1}+P^{2}
$$

Hence for the resolvent we obtain the equation

$$
v=R_{\tau} g \Longrightarrow P_{\tau} v=g
$$

By Duhamel's formula we also obtain a representation for the Fourier transform of the solution $v$ to the full forward Cauchy problem

$$
P v=f, \quad v(0)=h, v_{t}(0)=g
$$

Lemma 3.7. Let $v$ be the solution to 3.23 with $f \in L^{\infty} L^{2}, h \in \dot{H}^{1}$ and $g \in L^{2}$. Then for $\Im \tau<0$ we have

$$
\hat{v}(\tau)=R_{\tau}\left(\hat{f}(\tau)+\tau h+2 P_{1} h+g\right)
$$

This allows us to show that $P_{\tau}$ and $R_{\tau}$ are inverse operators if $\Im \tau<0$ :

Lemma 3.8. Let $\Im \tau<0$. Then $P_{\tau}: H^{2} \rightarrow L^{2}$ is a bounded one to one operator with dense range, and $R_{\tau}$ is its inverse. 
Proof. Given $u_{0} \in H^{2}$, apply the previous lemma to the function $u=u_{0} 1_{t>0}$ to see that $R_{\tau} P_{\tau} u_{0}=u_{0}$. The range is dense since it contains $H^{1}$. Indeed, by (3.22) and (3.20), for $f \in H^{1}$ we have $R_{\tau} f \in H^{2}$ and $P_{\tau} R_{\tau} f=f$.

\section{The RESOLVENT AND LOCAL ENERGY DECAY}

The goal of this section is to repeat the arguments of the previous section, but using the local energy decay property instead of the uniform boundedness. For this we consider the solution $u$ to the inhomogeneous forward problem

$$
P u=g \in L E^{*, k+4}, \quad k \geq 0
$$

with $g$ supported away from $t=-\infty$. From the local energy decay estimate (1.10) we obtain the weaker bound

$$
\|u\|_{L E^{1, k}} \leq c_{k}\|f\|_{L E^{*, k+3}}
$$

with a loss of three derivatives. We will use this bound to derive a similar resolvent bound. For each $\tau$ we use the $\mathcal{L} \mathcal{E}^{k}$ norms defined in (1.7), (1.8) to introduce the $\tau$ dependent norm $\mathcal{L} \mathcal{E}_{\tau}$ by

$$
\|v\|_{\mathcal{L E}_{\tau}^{k}}=\left\|\left(|\tau|+\langle r\rangle^{-1}\right) u\right\|_{\mathcal{L E}^{k}}+\|\nabla u\|_{\mathcal{L E}^{k}}+\left\|\left(|\tau|+\langle r\rangle^{-1}\right)^{-1} \nabla^{2} u\right\|_{\mathcal{L E}^{k}}
$$

Then we have

Proposition 4.9. Let $\Im \tau<0, k \geq 0, g \in \mathcal{L E}^{*, k+4}$ and $v=R_{\tau} g$. If the weak local energy decay bound (4.26) holds then we also have

$$
\|v\|_{\mathcal{L E}_{\tau}^{k}} \leq c_{k}\|g\|_{\mathcal{L E}^{*, k+4}}
$$

While this estimate is for now stated for $\Im \tau<0$, it is nevertheless uniform as $\tau$ approaches the real axis. Later we will use this fact to extend this estimate to all real $\tau$. If $-\Im \tau \gtrsim 1$ then this bound is superseded by (3.20). Thus only the case $-1<\Im \tau<0$ is of interest.

Proof. If we consider $f$ supported in a fixed dyadic region $A_{l}$ and measure $u$ in another region $A_{m}$, from (4.26) we obtain the uniform dyadic estimates

$$
\sum_{i \leq k} 2^{-\frac{m}{2}}\left\|\nabla_{x, t}^{i+1} u\right\|_{L^{2}\left(\mathbb{R} \times A_{m}\right)}+2^{-\frac{3 m}{2}}\left\|\nabla_{x, t}^{i} u\right\|_{L^{2}\left(\mathbb{R} \times A_{m}\right)} \lesssim \sum_{i \leq k+3} 2^{\frac{l}{2}}\left\|\nabla_{x, t}^{i} f\right\|_{L^{2}\left(\mathbb{R} \times A_{l}\right)}
$$

Since the equation (4.25) is solved forward in time, it is straightforward to add an exponentially decreasing weight in the above estimate. Precisely, from the above estimate one obtains a weighted version

$\sum_{i \leq k} 2^{-\frac{m}{2}}\left\|e^{-\epsilon t} \nabla_{x, t}^{i+1} u\right\|_{L^{2}\left(\mathbb{R} \times A_{m}\right)}+2^{-\frac{3 m}{2}}\left\|e^{-\epsilon t} \nabla_{x, t}^{i} u\right\|_{L^{2}\left(\mathbb{R} \times A_{m}\right)} \lesssim \sum_{i \leq k+3} 2^{\frac{l}{2}}\left\|e^{-\epsilon t} \nabla_{x, t}^{i} f\right\|_{L^{2}\left(\mathbb{R} \times A_{l}\right)}$

which holds uniformly with respect to $\epsilon>0$. If we further restrict $\epsilon$ to $0<\epsilon<1$ then we can uniformly move the exponential inside the differentiation to obtain

$$
\sum_{i \leq k} 2^{-\frac{m}{2}}\left\|\nabla_{x, t}^{i+1} e^{-\epsilon t} u\right\|_{L^{2}\left(\mathbb{R} \times A_{m}\right)}+2^{-\frac{3 m}{2}}\left\|\nabla_{x, t}^{i} e^{-\epsilon t} u\right\|_{L^{2}\left(\mathbb{R} \times A_{m}\right)} \lesssim \sum_{i \leq k+3} 2^{\frac{l}{2}}\left\|\nabla_{x, t}^{i} e^{-\epsilon t} f\right\|_{L^{2}\left(\mathbb{R} \times A_{l}\right)}
$$


Since we only have Hilbert space norms in the above estimate, we can take a Fourier transform and use Plancherel's theorem to obtain

$$
\begin{aligned}
& 2^{-\frac{m}{2}} \sum_{i \leq k}(1+|\tau|)^{k-i}\left(\left\|\nabla_{x}^{i+1} \hat{u}(\tau-i \epsilon)\right\|_{L^{2}\left(\mathbb{R} \times A_{m}\right)}+\left(2^{-m}+|\tau|\right)\left\|\nabla_{x}^{i} \hat{u}(\tau-i \epsilon)\right\|_{L^{2}\left(\mathbb{R} \times A_{m}\right)}\right) \\
& \lesssim \sum_{i \leq k+3} 2^{\frac{l}{2}}(1+|\tau|)^{k-i}\left\|\nabla_{x}^{i} \hat{f}(\tau-i \epsilon)\right\|_{L^{2}\left(\mathbb{R} \times A_{l}\right)}
\end{aligned}
$$

where the $L^{2}$ norms are now taken with respect to $\tau \in \mathbb{R}$ and $x \in \mathbb{R}^{3}$.

We recall that by 3.24 we have $\hat{u}(\tau-i \epsilon)=R_{\tau} \hat{f}(\tau-i \epsilon)$. Observing that the above bound holds for all $f$ in a dense subset of the Hilbert space defined by the norm on the right, it follows that in effect its pointwise version with respect to $\tau$ must also be valid, namely

$$
\begin{array}{r}
2^{-\frac{m}{2}} \sum_{i \leq k}(1+|\tau|)^{k-i}\left(\left\|\nabla_{x, t}^{i+1} v\right\|_{L^{2}\left(A_{m}\right)}+\left(2^{-m}+|\Re \tau|\right)\left\|\nabla_{x, t}^{i} v\right\|_{L^{2}\left(A_{m}\right)}\right) \\
\lesssim \sum_{i \leq k+3} 2^{\frac{l}{2}}(1+|\tau|)^{k-i}\left\|\nabla_{x, t}^{i} g\right\|_{L^{2}\left(A_{l}\right)}
\end{array}
$$

Here we have redenoted $\tau-i \epsilon$ by $\tau$, which now satisfies $\Im \tau<0$, and replaced $\hat{f}(\tau)$ by $g$ and $\hat{u}(\tau)$ by $v=R_{\tau} g$. We remark that in view of (3.20) we can replace the factor $|\Re \tau|$ by $|\tau|$ in the second term on the left.

Taking the suppremum over all $m \geq 0$, and then summing over all $l \geq 0$, we arrive at

$$
\sum_{i \leq k}(1+|\tau|)^{k-i}\left(\left\|\nabla_{x} v\right\|_{\mathcal{L E}^{i}}+\left\|\left(\langle r\rangle^{-1}+|\tau|\right) v\right\|_{\mathcal{L E}^{i}}\right) \lesssim \sum_{i \leq k+3}(1+|\tau|)^{k+3-i}\|g\|_{\mathcal{L E}^{*, i}}
$$

This concludes the transition from local energy decay to resolvent bounds. We remark that at the formal level one can go directly from (4.26) to 4.29) for real $\tau$ via Plancherel's theorem. However, in order to rigorously justify this analysis we are using the intermediate step of deriving uniform bounds for $\Im \tau<0$.

It remains to prove that (4.29) implies (4.28). This depends on the size of $\tau$. If $|\tau| \approx 1$ there is nothing to do. In the two remaining cases this transition is carried out via simple elliptic arguments.

(a) The case $|\tau| \ll 1$. Then we need to estimate the second derivative of $v$ for large $r$. We write the equation for $v$ in the form

$$
P^{2} v=-\tau^{2} v+i \tau P^{1} v
$$

This allows us to estimate

$$
\left\|\left(\langle r\rangle^{-1}+|\tau|\right)^{-1} P_{2} v\right\|_{\mathcal{L E}^{k}} \lesssim \tau\|v\|_{\mathcal{L} \mathcal{E}^{k}}+\left\|\nabla_{x} v\right\|_{\mathcal{L E}^{k}}+\|g\|_{\mathcal{L E}^{*}, k}
$$

where all the right hand side terms are already controlled by the right hand side in (4.26) via (4.29). Since $P^{2}$ is elliptic for large $r$, the transition from $P_{2} v$ bounds to $\nabla^{2} v$ bounds is done in a standard elliptic fashion within each dyadic region $A_{m}$.

(b) The case $|\tau| \gg 1$. The left hand side of (4.29) already contains the $\mathcal{L E}^{k}$ norm of $v$, we only need to be able to discard the $\tau$ factors on the right. For this we observe that the equation for $v$, namely

$$
\left(\tau^{2}+i \tau P^{1}+P^{2}\right) v=g
$$


is elliptic for low spatial frequencies $|\xi| \ll \tau$. This suggests we split $g$ into a low frequency and a high frequency part,

$$
g=S_{\leq \tau}\left(D_{x}\right) g+S_{\geq \tau}\left(D_{x}\right) g:=g_{\text {low }}+g_{\text {high }}
$$

For the high frequency part we can estimate

$$
\sum_{i \leq k+3}(1+|\tau|)^{k+3-i}\left\|g_{h i g h}\right\|_{\mathcal{L E}^{*, i}} \lesssim\|g\|_{\mathcal{L E}^{*, k+3}}
$$

and use (4.29) directly.

For the low frequency part, on the other hand, we reiterate the equation, setting

$$
v_{\text {low }}=\tau^{-2} g_{\text {low }}+v_{1}
$$

where $v_{1}$ solves

$$
P_{\tau} v_{1}=i \tau^{-1} P^{1} g_{\text {low }}+\tau^{-2} P^{2} g_{\text {low }}=g_{\text {low }, 1}
$$

Thus we have traded powers of $\tau$ for derivatives, which is favourable since $g_{\text {low }}$ is low frequency. Reiterating several times we arrive at

$$
v_{l o w}=\sum_{j=0}^{k-1} \tau^{-2-j} Q_{j} g_{l o w}+w_{l o w}
$$

where $Q_{j}$ are partial differential operators of order $j$ with smooth bounded coefficients and $w_{\text {low }}$ solves

$$
P_{\tau} w_{\text {low }}=\tau^{-k} Q_{k} g_{\text {low }}+\tau^{-k-1} Q_{k+1} g_{\text {low }}:=h_{\text {low }}
$$

The first term in $v_{\text {low }}$ is estimated directly in terms of $g_{\text {low }}$ while for $w_{\text {low }}$ we remark that

$$
\sum_{i \leq k+3}(1+|\tau|)^{k+3-i}\left\|h_{\text {low }}\right\|_{\mathcal{L E}^{*, i}} \lesssim\|g\|_{\mathcal{L E}^{*, k+3}}
$$

and use (4.29). The proof of the proposition is complete.

\section{VeCtor FIELD BOUNDS AND THE RESOLVENT LIMIT ON THE REAL AXIS}

Our first goal in this section is to prove further estimates for the operator $P_{\tau}$ by commuting it with several vector fields:

i) The rotations $\Omega=\left\{x_{i} \partial_{j}-x_{j} \partial_{i}\right\}$. Commuting them with $P_{\tau}$ we obtain

$$
\left[P_{\tau}, \Omega\right]=Q_{s r}
$$

where $Q_{s r}$ stands for a short range operator of the form

$$
Q_{s r}=\tau\left(h^{0 i} \partial_{i}+\partial_{i} h^{0 i}\right)+\partial_{i} h^{i j} \partial_{j}+h, \quad h^{0 i}, h^{i j} \in l^{1} S\left(r^{-1}\right), \quad h \in l^{1} S\left(r^{-3}\right)
$$

ii) The scaling $S=-\tau \partial_{\tau}+r \partial_{r}$. Then the commutator is

$$
\left[P_{\tau}, S\right]=2 P_{\tau}+Q_{l r}+Q_{s r}
$$

where the long range component is radial and has the form

$$
Q_{l r}=k^{\omega} \Delta_{\omega}+k, \quad k^{\omega}, k \in S_{r a d}\left(r^{-3}\right)
$$


The next proposition applies for spatial functions $g$, in which case $S=S_{r} g$. However, it is also interesting to allow $g$ to depend on the parameter $\tau$.

Proposition 5.10. Let $\Im \tau<0$ and $g \in \mathcal{L} \mathcal{E}^{*}$, possibly depending on $\tau$, so that

$$
\left\|T^{i} \Omega^{j} S^{k} g\right\|_{\mathcal{L E}^{*}} \leq 1, \quad i+4 j+16 k \leq N
$$

Then

$$
\left\|T^{i} \Omega^{j} S^{k} R_{\tau} g\right\|_{\mathcal{L} \mathcal{E}_{\tau}} \lesssim 1, \quad i+4 j+16 k \leq N-4
$$

Proof. Set $v=R_{\tau} g$. To illustrate the method we first consider the simplest cases. In the case of $\Omega$ we have

$$
P_{\tau} \Omega v=\Omega g+Q_{s r} v
$$

A direct computation shows that

$$
\left\|Q_{s r} v\right\|_{\mathcal{L E}^{*, k}} \lesssim\|v\|_{\mathcal{L E}_{\tau}^{k}}
$$

Hence by (4.28) we obtain

$$
\|\Omega v\|_{\mathcal{L E}_{\tau}^{k}} \lesssim\|\Omega g\|_{\mathcal{L E}^{*, k+4}}+\|g\|_{\mathcal{L E}^{*, k+8}}
$$

Consider now the operator $\Omega^{2}$. We have

$$
P_{\tau} \Omega^{2} v=\Omega^{2} f+Q_{s r} \Omega v+Q_{s r} v
$$

so we conclude again via (5.32) and (4.28) that

$$
\left\|\Omega^{2} v\right\|_{\mathcal{L} \mathcal{E}_{\tau}^{k}} \lesssim\left\|\Omega^{2} g\right\|_{\mathcal{L} \mathcal{E}^{*, k+4}}+\|\Omega g\|_{\mathcal{L} \mathcal{E}^{*, k+8}}+\|g\|_{\mathcal{L} \mathcal{E}^{*, k+12}}
$$

Next consider the scaling field $S$. We have

$$
P_{\tau} S v=S g+2 g+Q_{s r} v+Q_{l r} v
$$

For $Q_{s r}$ we use (5.32), while for $Q_{l r}$ we use

$$
\left\|Q_{l r} v\right\|_{\mathcal{L} \mathcal{E}^{*, k}} \lesssim\|v\|_{\mathcal{L E}_{\tau}^{k}}+\left\|\Omega^{2} v\right\|_{\mathcal{L E}_{\tau}^{k}}
$$

to obtain

$$
\|S v\|_{\mathcal{L E}_{\tau}^{k}} \lesssim\|S g\|_{\mathcal{L E}^{*, k+4}}+\left\|\Omega^{2} g\right\|_{\mathcal{L E}^{*, k+8}}+\|\Omega g\|_{\mathcal{L E}^{*, k+12}}+\|g\|_{\mathcal{L} \mathcal{E}^{*, k+16}}
$$

The proof of the general case is by induction. The details are left for the reader.

Now we have enough information in order to define the pointwise limit of the resolvent as $\tau$ approaches the real line. The next result deals with the limit on $\mathbb{R} \backslash\{0\}$. The detailed analysis near frequency zero is left for the next section.

Proposition 5.11. i) The operators $R_{\tau}$ extend continuously from the lower half-space to the real line $\mathbb{R} \backslash\{0\}$ in the $H_{\text {comp }}^{4} \rightarrow L_{\text {loc }}^{2}$ topology, and the bound (4.28) holds uniformly for all real $\tau$.

ii) Let $\tau \in \mathbb{R} \backslash\{0\}$ and $g \in \mathcal{L E}^{*, 4}$. Then the function $v=R_{\tau} g$ satisfies the outgoing radiation condition

$$
\lim _{j \rightarrow \infty} 2^{-\frac{j}{2}}\left\|\left(\partial_{r}+i \tau\right) v\right\|_{L^{2}\left(A_{j}\right)}=0
$$


iii) Conversely, suppose that $\tau \in \mathbb{R} \backslash 0$, and $v \in \mathcal{L E}_{\tau}^{4}$ which satisfies the outgoing radiation condition (5.34). If $P_{\tau} v=g \in \mathcal{L E}^{*, 4}$ then $v=R_{\tau} g$.

This result allows us to transfer the previous bounds in this section from the lower half-plane to the real axis.

Corollary 5.12. The results in Propositions 4.95 .10 and Proposition 6.15 apply for all nonzero real $\tau$. In addition, for $i, j, k$ as in Proposition [5.10, the functions $T^{i} \Omega^{j} S^{k} R_{\tau} g$ satisfy the outgoing radiation condition (5.34).

Proof. i) In view of the uniform bound (4.28) it suffices to establish the convergence on a dense subset. Let $g \in \mathcal{L E}^{*}$ so that (5.30) holds for some large $N$. Then by (5.31) we have

$$
\left\|S R_{\tau} g\right\|_{\mathcal{L E}} \lesssim 1
$$

Hence we can write

$$
\tau \partial_{\tau} R_{\tau} g=S R_{\tau} g+r \partial_{r} R_{\tau}
$$

In view of the $\mathcal{L} \mathcal{E}_{\tau}$ bounds for $R_{\tau} g$ and $S R_{\tau} g$ we can bound the LHS locally in $L^{2}$ and in $H^{1}$. Hence the map $\tau \rightarrow R_{\tau} g$ is locally Lipschitz away from $\tau=0$ from $\{\Im \tau \leq 0\}$ into $H_{l o c}^{1}$. The desired convergence follows.

ii) By (4.28) it suffices to establish the radiation condition (5.34) for $g$ in a dense subset of $\mathcal{L E}^{*, 4}$. Here we consider functions $g \in \mathcal{L E}^{*}$ so that (5.30) holds for $g$ for some large $N$, and so that in addition $g$ decays one order faster at infinity, $\langle r\rangle g \in \mathcal{L E}^{*}$. We denote $v(\tau)=R_{\tau} g$.

For some large $R$ we truncate $v(\tau)$ to the exterior of a ball of size $R$ and set

$$
w(\tau)=\chi_{>R} v(\tau) .
$$

This does not affect its behavior at spatial infinity and thus the radiation condition. The functions $w(\tau)$ solve an equation of the form

$$
\left(\Delta+\tau^{2}\right) w=Q_{l r} w+Q_{s r} w+\left[P_{\tau}, \chi>R\right] v(\tau)+\chi>R g:=h(\tau)
$$

We can now use the local energy decay estimate for $\Delta+\tau^{2}$, namely

$$
\|\nabla w\|_{\mathcal{L E}}+\left\|\left(|\tau|+\langle r\rangle^{-1}\right) w\right\|_{\mathcal{L E}} \lesssim\|h\|_{L E^{*}}
$$

which can be either proved directly or derived from (1.9) for $\square$ in the same way (4.28) was obtained from (1.10).

In view of (5.31) and of the condition $\langle r\rangle g \in \mathcal{L E}^{*}$ we can estimate uniformly for $\tau$ in a compact subset of $\{\Im \tau \leq 0\} \backslash\{0\}$

$$
\|\langle r\rangle h(\tau)\|_{\mathcal{L E}^{*}} \lesssim 1
$$

The radiation condition follows if we can show that the solutions $w(\tau)$ to (5.35) satisfy

$$
\left\|\langle r\rangle\left(\partial_{r}+i \tau\right) w\right\|_{\mathcal{L E}} \lesssim\|\langle r\rangle h\|_{\mathcal{L} \mathcal{E}^{*}}
$$

uniformly on compact sets. Indeed, let us estimate $w$ in a dyadic region $A_{m}$. We split $h=\chi_{<m-2} h+\chi_{\geq m-2} h$. We have

$$
\|\chi \geq m-2,\|_{\mathcal{L E}^{*}} \lesssim 2^{-m}\|\langle r\rangle h\|_{\mathcal{L} \mathcal{E}^{*}}
$$


therefore by (5.36) we have

$$
\left\|\chi_{m}\left(\partial_{r}+i \tau\right)\left(\Delta+\tau^{2}\right)^{-1} \chi_{\geq m-2} h\right\|_{\mathcal{L E}} \lesssim 2^{-m}\|\langle r\rangle h\|_{\mathcal{L E}^{*}}
$$

It remains to prove the same bound for the other component $\chi_{<m-2} h$. For this use the kernel for the operator $\left(\Delta+\tau^{2}\right)^{-1}$ which is

$$
K_{\tau}(y)=|y|^{-1} e^{-i \tau|y|}
$$

This is justified for $\Im \tau<0$, where the symbol of $\Delta+\tau^{2}$ is nonzero, and in effect bounded from below. A direct computation gives

$$
\left(\partial_{r}+i \tau\right)\left(\Delta+\tau^{2}\right)^{-1} h(x)=e^{-i \tau|x|} \int h(y)|x-y|^{-1} e^{-i \tau|x-y|} a(x, y) d y
$$

where

$$
a(x, y)=-\frac{x \cdot(x-y)}{|x||x-y|^{2}}-i \tau\left(\frac{x \cdot(x-y)}{|x||x-y|}-1\right)
$$

We are interested in the case where we have the localization $|x| \approx 2^{m}$ and $2|y| \leq|x|$. There we have $|a| \lesssim 2^{-m}\langle y\rangle$. Taking absolute values inside the integral, we use again (5.36) with $\tau=0$ to obtain

$$
\left\|\chi_{m}\left(\partial_{r}+i \tau\right)\left(\Delta+\tau^{2}\right)^{-1} \chi_{<m-2} h\right\|_{\mathcal{L E}} \lesssim 2^{-m}
$$

The proof of (5.37) is concluded.

iii) If $v$ is compactly supported then for $\epsilon>0$ we have

$$
v=R_{\tau-i \epsilon} P_{\tau-i \epsilon} v
$$

But the right hand side converges to $R_{\tau} g$ locally in $L^{2}$, and the proof is concluded.

Suppose now that $v$ is supported away from 0 . We first use the radiation condition for $v$ to derive a similar radiation condition for its derivatives,

$$
\lim _{j \rightarrow \infty} 2^{-\frac{j}{2}}\left\|\nabla^{k}\left(\partial_{r}+i \tau\right) v\right\|_{L^{2}\left(A_{j}\right)}=0, \quad k \leq 4
$$

We recall that $P_{\tau}$ has the form

$$
P_{\tau}=\Delta+\tau^{2}+P_{l r}+P_{s r},
$$

therefore by commuting and estimating all terms with decaying coefficients in terms of $v$ we obtain

$$
\left\|\langle r\rangle^{-1}\left(\Delta+\tau^{2}\right)\left(\partial_{r}+i \tau\right) v\right\|_{\mathcal{L E}^{*, 4}} \lesssim\|v\|_{\mathcal{L E}_{\tau}^{4}}+\|g\|_{\mathcal{L} \mathcal{E}^{*, 4}}
$$

Hence from the radiation condition (5.34) it follows that

$$
\lim _{j \rightarrow \infty} 2^{-\frac{j}{2}}\left\|\Delta\left(\partial_{r}+i \tau\right) v\right\|_{L^{2}\left(A_{j}\right)}=0
$$

Combined with (5.34) and elliptic estimates for $\Delta$ we obtain a similar decay property for $\nabla\left(\partial_{r}+i \tau\right)$ and $\nabla^{2}\left(\partial_{r}+i \tau\right)$. Reiterating we arrive at (5.38).

For $\epsilon>0$ we define the functions $v_{\epsilon}=v e^{-\epsilon\langle r\rangle}$ which satisfy $v_{\epsilon}=R_{\tau-i \epsilon} P_{\tau-i \epsilon} v_{\epsilon}$. By (4.28) and part (i) of the proposition, the desired conclusion would follow if we are able to show that

$$
P_{\tau-i \epsilon} v_{\epsilon} \rightarrow g \quad \text { in } \mathcal{L E}^{*, 4}
$$


For this we use (5.39) to compute directly

$P_{\tau-i \epsilon} v_{\epsilon}=g e^{-\epsilon\langle r\rangle}+2 \epsilon\left(\partial_{r}+i \tau+\frac{1}{r}\right) v e^{-\epsilon\langle r\rangle}+\left(\epsilon l^{1} S\left(r^{-2}\right)+\epsilon^{2} l^{1} S\left(r^{-1}\right)+\epsilon l^{1} S\left(r^{-1}\right) \nabla\right) v e^{-\epsilon\langle r\rangle}$

Since $g \in \mathcal{L E}^{*, 4}$, the first term converges to $g$ in $\mathcal{L E}^{*, 4}$. For the $\left(\partial_{r}+i \tau\right) v$ part of the second term, the same follows from the expanded radiation condition (5.38). Finally for the remaining terms we obtain $O(\epsilon)$ decay in $\mathcal{L E}^{*, 4}$ from the $\mathcal{L E}_{\tau}^{4}$ bound for $v$. Thus (5.40) follows and the proof is concluded.

\section{The RESOlVEnt ANALysis NEAR ZERO FREQUenCy}

In this section we first study the limit of the resolvent at frequency $\tau=0$. Then we consider data with more regularity and decay at spatial infinity, and we produce an expansion of the zero resolvent in terms of powers of $r$. Finally, we obtain a quadratic expansion of the resolvent near frequency 0 for data with faster decay. We begin by establishing the existence of the limit of the resolvent at zero.

Proposition 6.13 (Resolvent extension to $\tau=0$ ). a) Let $g,\langle r\rangle g \in \mathcal{L E}^{*, 4}$. Then the limit

$$
R_{0} g=\lim _{\epsilon \rightarrow 0} R_{-i \epsilon} g
$$

exists in the $L_{l o c}^{2}$ topology, and the following bound holds:

$$
\left\|\langle r\rangle R_{0} g\right\|_{\mathcal{L E}_{0}^{k}} \lesssim\|\langle r\rangle g\|_{\mathcal{L E}^{*, 4+k}}, \quad k \geq 0
$$

b) The operator $R_{0}$ admits a unique continuous extension to $\mathcal{L E}^{*, 4}$ which satisfies

$$
\left\|R_{0} g\right\|_{\mathcal{L E}_{0}^{k}} \lesssim\|g\|_{\mathcal{L E}^{*, 4+k}}, \quad k \geq 0
$$

respectively

$$
\lim _{j \rightarrow \infty} 2^{\left(m-\frac{3}{2}\right) j}\left\|\nabla^{m} R_{0} g\right\|_{L^{2}\left(A_{j}\right)}=0, \quad j=0,1,2
$$

c) Conversely, let $v \in \mathcal{L} \mathcal{E}_{0}^{4}$ so that $P_{0} v=g \in \mathcal{L E}^{*, 4}$ and

$$
\lim _{j \rightarrow \infty} 2^{-\frac{j}{2}}\left\|\partial_{r} v\right\|_{L^{2}\left(A_{j}\right)}=0 .
$$

Then $v=R_{0} g$.

Proof. (a), the case $k=0$. Denote $v_{\epsilon}=R_{-i \epsilon} g$. By the local energy decay estimate (4.28) we know that

$$
\left\|v_{\epsilon}\right\|_{\mathcal{L E}_{i \epsilon}} \lesssim 1
$$

This already suffices in order to obtain the existence of a strong limit $v_{\epsilon} \rightarrow v$ in $H_{l o c}^{1}$ on a subsequence. To prove convergence it suffices to establish the uniqueness of the limit. For this we first study the regularity of the limit. A-priori we know that $v \in \mathcal{L} \mathcal{E}_{0}$, but this does not suffice for uniqueness.

The equation for $v_{\epsilon}$ has the form $P_{-i \epsilon} v_{\epsilon}=g$, or in expanded form

$$
\left(P^{2}-\epsilon P^{1}-\epsilon^{2}\right) v_{\epsilon}=g
$$


with $P^{2}$ selfadjoint and $P^{1}$ skew-adjoint. Since $v_{\epsilon} \in H^{2}$, we cam multiply by $v_{\epsilon}$ and integrate by parts to obtain

$$
\Re \int g v_{\epsilon} d x=\int-P^{2} v_{\epsilon} \cdot v_{\epsilon}+\epsilon^{2} v_{\epsilon}^{2} d x
$$

The principal part of $-P_{2}$ is positive definite outside a compact set $K$, so we obtain

$$
\left\|\nabla v_{\epsilon}\right\|_{L^{2}}^{2} \lesssim\left|\int g v_{\epsilon} d x\right|+\int_{K}\left|\nabla v_{\epsilon}\right|^{2} d x+\int\langle r\rangle^{-3}\left|v_{\epsilon}\right|^{2} d x
$$

For the first term on the right we use the fact that $\langle r\rangle g \in \mathcal{L E}^{*}$ and $\langle r\rangle^{-1} v_{\epsilon} \in \mathcal{L} \mathcal{E}$. For the second term on the right we use the $\mathcal{L} \mathcal{E}_{i \epsilon}$ bound for $v_{\epsilon}$. In the last term we use the Hardy inequality to absorb the far away part into the left hand side, and the $\mathcal{L E}_{i \epsilon}$ norm for the near part. We obtain an uniform bound

$$
\left\|\nabla v_{\epsilon}\right\|_{L^{2}}^{2} \lesssim\|\langle r\rangle g\|_{\mathcal{L E}^{*, 4}}
$$

so any limit $v$ of a subsequence must belong to $\dot{H}^{1}$. Suppose we have two distinct limit points $v_{1}, v_{2}$. The difference $v=v_{1}-v_{2}$ must satisfy $v \in \dot{H}^{1}, P_{0} v=0$. But this would contradict the local energy decay estimates for the function $v 1_{t \geq 0}$ which solves the homogeneous wave equation in $[0, \infty)$.

It remains to boost the decay of $v$ and its derivatives near infinity to $\langle r\rangle v \in \mathcal{L} \mathcal{E}_{0}$. For this we view $P_{0}$ as a perturbation of $-\Delta$ near infinity. Precisely, for large $R$ we consider the equation for $w=\chi>R v$,

$$
P_{0} w=\chi_{>R}(r) g+\left[P_{0}, \chi_{>R}(r)\right] v:=h
$$

Since the above commutator has compact support, we can estimate

$$
\|\langle r\rangle h\|_{\mathcal{L} \mathcal{E}^{*}} \lesssim\|\langle r\rangle g\|_{\mathcal{L} \mathcal{E}^{*}}+\|v\|_{\mathcal{L E}_{0}} \lesssim\|g\|_{\mathcal{L E}^{*, 4}}
$$

We rewrite the above equation as

$$
-\Delta w=h+\chi_{>R / 2}(r)\left(P_{l r}+P_{s r}\right) w
$$

We solve this last equation via a Picard iteration. It is easy to verify directly that

$$
\left\|\langle r\rangle(-\Delta)^{-1} h\right\|_{\mathcal{L} \mathcal{E}_{0}} \lesssim\|\langle r\rangle h\|_{\mathcal{L E}^{*}}
$$

where $(-\Delta)^{-1}$ is the convolution operator with $r^{-1}$.

On the other hand the last term on the right is perturbative for large $R$,

$$
\left\|\langle r\rangle \chi_{>R / 2}(r)\left(P_{l r}+P_{s} r\right) w\right\|_{\mathcal{L E}^{*}} \lesssim R^{-1}\|\langle r\rangle w\|_{\mathcal{L E}_{0}}
$$

Thus a unique solution $w \in\langle r\rangle^{-1} \mathcal{L E}_{0} \subset \dot{H}^{1}$ is found iteratively. On the other hand, by energy estimates as above the equation (6.45) also has a unique solution in $\dot{H}^{1}$, therefore the $\langle r\rangle^{-1} \mathcal{L} \mathcal{E}_{0}$ coincides with the $\dot{H}^{1}$ solution constructed from $v$. This proves the existence of the limit of $u_{\epsilon}$ as $\epsilon \rightarrow 0$, as well as 6.41) for $k=0$.

(b), proof of (6.42). The bound 6.42) is obtained by passing to the limit $\tau=i \epsilon \rightarrow 0$ in the estimate (4.28). Since $\langle r\rangle^{-1} \mathcal{L E}^{*, 4}$ is dense in $\mathcal{L E}^{*, 4}$, this provides us with an unique continuous extension which satisfies the bound (6.42).

(a), the case $k>0$. Now (6.41) for $k \geq 1$ follows by combining the regularity of $v$ provided by (6.42) for small $r$ with the decay at infinity given by the $k=0$ case of (6.41) 
plus elliptic regularity for large $r$. The reason we need to argue in this roundabout way is that $P_{0}$ is elliptic for large $r$, but not necessarily for small $r$.

(b), proof of (6.43). By (6.42), it suffices to establish (6.43) for $g$ in a dense subset of $\mathcal{L E}^{*, 4+k}$. But this is provided by (6.41).

(c). This proof is identical to the proof of Proposition 5.11 (iii). First the higher regularity version of (6.44) are established, namely

$$
\lim _{j \rightarrow \infty} 2^{-\frac{j}{2}}\left\|\nabla^{k} \partial_{r} v\right\|_{L^{2}\left(A_{j}\right)}=0, \quad k \leq 4 .
$$

Then the relation $v=P_{0} g$ is obtained as the limit of $v_{\epsilon}=R_{-i \epsilon} P_{-i \epsilon} v_{\epsilon}$ for $v_{\epsilon}=v e^{-\epsilon r}$.

Our next result provides better bounds for $R_{0}$ on functions with more regularity and decay.

Proposition 6.14 (Higher regularity for $R_{0}$ ). a) If $g \in Z^{k+4,1}$ then the following representation holds for large $r$

$$
R_{0} g=c(r)\langle r\rangle^{-1}+v_{1}
$$

where, with $N$ arbitrarily large, we have the bounds

$$
\|c\|_{L^{\infty}}+\left\|S_{r} c\right\|_{l^{1} S(1)}+\left\|v_{1}\right\|_{Z^{k,-1}} \lesssim\|g\|_{Z^{k+4,1}}
$$

b) If we further have $g \in Z^{k+4,2}$ then $R_{0} f$ admits the large $r$ representation

$$
R_{0} g=c\langle r\rangle^{-1}+d(r) \cdot \nabla\langle r\rangle^{-1}+e(r)\langle r\rangle^{-2}+v_{2}
$$

where, with $N$ arbitrarily large, we have the bounds

$$
|c|+\|d\|_{L^{\infty}}+\left\|S_{r} d\right\|_{l^{1} S(1)}+\|e\|_{S(1)}+\left\|v_{2}\right\|_{Z^{k, 0}} \lesssim\|g\|_{Z^{k+4,2}}
$$

Here, by a slight abuse of notation, by the $S(1)$ and $l^{1} S(1)$ norm we understand the sum of an arbitrarily large but finite number of seminorms for the respective symbol classes.

We note the key presence of the $e(r) r^{-2}$ term in (6.48), which is the highest order contribution due to the long range scalar potential. This term will later turn out to produce the first nonanalytic contribution in the expansion of $R_{\tau}$ with respect to $\tau$ near $\tau=0$. Thus this term ultimately determines the final decay rate in our main theorem.

Proof. a) Consider (6.46). For the local (small $r$ ) regularity of $v$ we only need the bound (4.28). Thus it suffices to prove the same estimate for the solution $w$ to the near infinity problem 6.45). We begin with the simpler case $h \in\langle r\rangle^{-1} \mathcal{L E}^{*}$, in which case we seek to prove that $w$ admits a representation as in (6.46) so that

$$
\|c\|_{L^{\infty}}+\left\|S_{r} c\right\|_{l^{1} S(1)}+\sum_{j \leq 2}\left\|\langle r\rangle^{-1+j} \nabla_{x}^{j} v_{1}\right\|_{\mathcal{L E}^{*}} \lesssim\|\langle r\rangle h\|_{\mathcal{L E}^{*}}
$$

The second right hand side term in (6.45) is perturbative with respect to this estimate with a small $O\left(R^{-1}\right)$ bound, so it suffices to prove that this representation is valid for the simpler equation

$$
-\Delta w=h
$$


Then $w$ is computed using the fundamental solution for $-\Delta$ in $\mathbb{R}^{3}$, namely

$$
w=h * r^{-1}
$$

We consider a dyadic decomposition of $h$ with respect to the $\left\{A_{m}\right\}$ partition of $\mathbb{R}^{3}$,

$$
h=\sum_{m} h_{m}
$$

Correspondingly we partition $w$ into

$$
w=\sum_{m} w_{m}^{l o w}+w_{m}^{h i g h}, \quad w_{m}^{l o w}=\chi_{<m+2}\left(h_{m} * r^{-1}\right)
$$

For the first part we directly estimate in a scale invariant elliptic fashion

$$
2^{-2 m}\left\|w_{m}^{\text {low }}\right\|_{L^{2}}+2^{-m}\left\|\nabla w_{m}^{\text {low }}\right\|_{L^{2}}+\left\|\nabla^{2} w_{m}^{\text {low }}\right\|_{L^{2}} \lesssim\left\|h_{m}\right\|_{L^{2}}
$$

Hence is easy to see that the sum of the $w_{m}^{\text {low }}$ contributions can be included in $v_{1}$.

Consider now the functions $w_{m}^{h i g h}$, which we expand near infinity as

$$
w_{m}^{h i g h}=c_{m} \chi>m+2|x|^{-1}+z_{m}
$$

where

$$
c_{m}=\int h_{m}(y) d y, \quad z_{m}=\chi_{>m+2} \int h_{m}(y)\left(\frac{1}{|x-y|}-\frac{1}{|x|}\right) d y .
$$

By Cauchy-Schwarz we can estimate

$$
\left\|c_{m}\right\|_{l^{1}} \lesssim\|\langle r\rangle h\|_{\mathcal{L E}^{*}}
$$

therefore the first term in (6.51) yields the first term in (6.46), with

$$
c(r)=\sum_{m} c_{m} \chi>m+2
$$

The second term in (6.51), on the other hand, can be incorporated into $v_{1}$. Indeed we can bound

$$
\left|\frac{1}{|x-y|}-\frac{1}{|x|}\right| \lesssim \frac{|y|}{|x|^{2}}, \quad|x|>2|y|
$$

which yields the off-diagonal decay

$$
\left\|\langle r\rangle^{j} \nabla^{j} z_{m}\right\|_{L^{\infty}\left(A_{n}\right)} \lesssim 2^{m-n}, \quad m \geq n+2
$$

which suffices after summation with respect to $n$ and $m$. This concludes the proof of (6.50). The more general bound 6.47) follows from 6.50 by elliptic regularity once we observe that $-\Delta\left(c(r) r^{-1}\right) \in l^{1} S\left(r^{-2}\right)$.

b) Finally we consider the case when $g \in Z^{k+4,2}$. Again it suffices to prove the same estimate for the solution $w$ to the near infinity problem (6.45). In a manner similar to the previous case, we will first construct a decomposition (6.48) of $w$ so that the following estimate holds:

$|c|+\|d\|_{L^{\infty}}+\left\|S_{r} d\right\|_{l^{1} S(1)}+R^{-\frac{1}{2}}\|e\|_{S(1)}+\sum_{j \leq 2}\left\|\langle r\rangle^{2-j} \nabla_{x}^{j} v_{2}\right\|_{\mathcal{L E}^{*}} \lesssim\|h\|_{\langle r\rangle^{-2} \mathcal{L E}^{*}+R^{\frac{1}{2}} S_{\text {rad }}\left(r^{-4}\right)}$

Once this is done, its higher regularity version (6.49) follows by elliptic theory.

We remark that in the above estimate we have harmlessly added an $S_{\text {rad }}\left(r^{-4}\right)$ component to $h$. Its purpose is to render the second right hand side term in 6.45) fully 
perturbative. The $R^{ \pm \frac{1}{2}}$ weights serve the same goal. To understand how this works we split $w$ into $w=c\langle r\rangle^{-1}+w_{1}$, and consider all possible terms:

i) The output of both the short range operator $P_{s r}$ and the long range operator $P_{l r}$ applied to $w_{1}$ can be estimated as follows:

$\left\|\chi>R\left(P_{s r}+P_{l r}\right) w_{1}\right\|_{\langle r\rangle^{-2} \mathcal{L} \mathcal{E}^{*}} \lesssim R^{-1}\left(\|d\|_{L^{\infty}}+\left\|S_{r} d\right\|_{l^{1} S(1)}+\|e\|_{S(1)}+\sum_{j \leq 2}\left\|\langle r\rangle^{2-j} \nabla_{x}^{j} v_{2}\right\|_{\mathcal{L E}^{*}}\right)$

which provides an $R^{-\frac{1}{2}}$ gain compared with (6.52).

ii) The output of the short range operator $P_{s r}$ applied to $c(r)\langle r\rangle^{-1}$ satisfies the weaker bound

$$
\left\|P_{s r} c\langle r\rangle^{-1}\right\|_{l^{1} S\left(r^{-4}\right)} \lesssim|c|
$$

which gives decay when restricted to $r \gtrsim R$,

$$
\left\|P_{s r} c\langle r\rangle^{-1}\right\|_{\langle r\rangle^{-2} \mathcal{L E}} \lesssim o_{R}(1)|c|,
$$

where the decay of the constant as $R \rightarrow \infty$ comes from the fact that the $l^{1}$ summation is restricted to dyadic regions $A_{m}$ with $2^{m} \gtrsim R$.

iii) The output of the $k^{\omega}(r) \Delta_{\omega}$ part of $P_{l r}$ applied to $c(r)\langle r\rangle^{-1}$ is zero.

iv) Finally he output of the $k(r)$ part of $P_{l r}$ applied to $c(r)\langle r\rangle^{-1}$ belongs to $S\left(r^{-4}\right)$, and we have an $R^{-\frac{1}{2}}$ gain compared to (6.52) due to the choice of constants in (6.52).

Thus we need to prove the bound (6.52) for an expansion (6.49) of the solution $w$ to the equation

$$
-\Delta w=h, \quad \operatorname{supp} h \subset\{r \gtrsim R\}
$$

We consider first the case when $\langle r\rangle^{2} h \in \mathcal{L E}^{*}$. We proceed as in the proof of (6.46)(6.47). The analysis of $w_{m}^{l o w}$ rests unchanged. However, for $w_{m}^{\text {high }}$ we need a second order expansion at infinity, namely

$$
w_{m}^{h i g h}=c_{m} \chi_{>m+2}|x|^{-1}+d_{m}^{i} \chi_{>m+2} \partial_{i} r^{-1}+z_{m},
$$

where

$$
\begin{gathered}
c_{m}=\int h_{m}(y) d y, \quad d_{m}^{i}=\int y_{i} h_{m}(y) d y \\
z_{m}=\chi_{>m+2} \int h_{m}(y)\left(\frac{1}{|x-y|}-\frac{1}{|x|}-\frac{y \cdot x}{|x|^{2}}\right) d y .
\end{gathered}
$$

Then we estimate

$$
2^{m}\left|c_{m}\right|+\left|d_{m}^{i}\right|+\left\|z_{m}\right\|_{Z^{N, 0}} \lesssim\left\|\langle r\rangle^{2} h_{m}\right\|_{\mathcal{L E}^{*}}
$$

Hence we can set

$$
c=\sum_{m} c_{m}, \quad d^{i}(r)=\sum_{m} d_{m}^{i} \chi_{>m+2}(r), \quad e(r)=r \sum_{m} c_{m} \chi_{<m+2}(r)
$$

One easily checks that $c, d^{i}$ and $e$ satisfy bounds as in (6.52), so the proof of (6.52) is concluded in this case.

It remains to consider the case when $h \in S_{\text {rad }}\left(r^{-4}\right)$. Then $w$ is also smooth and spherically symmetric, and we can write

$$
\partial_{r}^{2}(r w)=r h
$$


Integrating this relation twice, first from infinity and the second time from zero, it follows that $w$ has the form

$$
w_{2}=c\langle r\rangle^{-1}+e(r)\langle r\rangle^{-2}, \quad e \in S_{\text {rad }}(1)
$$

Furthermore, since $h$ is supported in $r \gtrsim R$, we obtain

$$
|c| \lesssim R^{-1}
$$

Thus the proof of (6.52) for $w$ is concluded.

The next step is to use the $R_{0}$ estimates in order to improve the analysis of $R_{\tau} g$ for $\tau$ near 0 , and in particular the bounds in Proposition 5.10, in the case when $g$ has better decay at infinity. Precisely, as in the previous proposition, we will successively consider the case when $g \in Z^{k+4,1}$ and when $g \in Z^{k+4,2}$. We will use the notation $a \wedge b$ for a smooth 1-homogeneous function of $a, b \in \mathbb{R}^{+}$which equals $\min \{a, b\}$ unless $a \approx b$.

Proposition 6.15. a) Let $g \in Z^{k+4,1}$ and $|\tau| \lesssim 1$ with $\Im \tau \leq 0$. Then the function $v(\tau)=R_{\tau} g$ can be represented as

$$
v(\tau)=e^{-i \tau\langle r\rangle} v(0)+w_{1}(\tau)
$$

where the function $w_{1}$ is given by

$$
w_{1}(\tau)=R_{\tau}\left(\chi_{>|\tau|^{-1}}(r) g+\tau h\right)
$$

with $h$ satisfying

$$
\left\|r^{m}\left(\partial_{r}+i \tau\right)^{m} T^{i} \Omega^{j} S^{l} h\right\|_{\mathcal{L} \mathcal{E}^{*}} \lesssim 1, \quad m+i+j+l \leq k
$$

b) Assume in addition that $g \in Z^{k+4,2}$. Then $v$ can be represented as

$$
v(\tau)=\left(v(0)+\tau v_{1}+\tau e_{0}(r, \tau)\right) e^{-i \tau r}+w_{2}(\tau)
$$

where

the radial function $e_{0}$ has the form

$$
v_{1}=R_{0} g_{1}, \quad g_{1} \in Z^{k, 1},
$$

$$
e_{0}(r, \tau)=r^{-1} e_{1}\left(r \wedge|\tau|^{-1}\right)+\tau\left(e_{2}\left(r \wedge|\tau|^{-1}\right)-e_{2}\left(|\tau|^{-1}\right)\right), \quad e_{1}, e_{2} \in S(\log r)
$$

and $w_{2}$ is given by

$$
w_{2}(\tau)=R_{\tau}\left(\chi_{>|\tau|^{-1}} g+\tau^{2} h\right)
$$

with $h$ satisfying (6.54).

Proof. a) We note that the function $e^{-i \tau\langle r\rangle} v(0)$ belongs to the range of $R_{\tau}$ since it satisfies the outgoing radiation condition (5.34). We compute the equation for the function $w_{1}=$ $v(\tau)-e^{-i \tau r} v(0)$ :

$$
\begin{aligned}
P_{\tau} w_{1}= & g+\left(g e^{-i \tau\langle r\rangle}-g\right)+2 \tau\left(\partial_{r}+\frac{1}{r}\right) v(0) e^{-i \tau\langle r\rangle} \\
& +\left(\tau l^{1} S\left(r^{-2}\right)+\tau^{2} l^{1} S\left(r^{-1}\right)+\tau l^{1} S\left(r^{-1}\right) \nabla\right) v(0) e^{-i \tau\langle r\rangle}
\end{aligned}
$$

where the second term on the right comes from the Laplacian and the third is generated by the short range part of $P$. We observe that the long range part of $P$ does not produce any contributions because the function $e^{i \tau\langle r\rangle}$ is radial. 
For $v(0)$ we use the representation (6.46) and the corresponding bounds 6.47). This allows us to estimate all terms on the right but the first one as in (6.54). We note in particular that in order to estimate the contribution of the first term in (6.46) to the second term above, it is necessary to take advantage of the improved $l^{1} S(1)$ bound on $S_{r} c$ in 6.47).

Finally we consider the first term on the right in (6.55), which we rewrite as

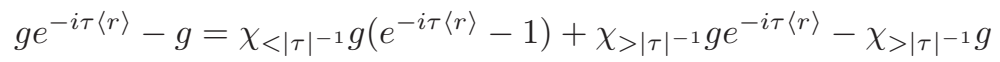

In the first term we can pull out a $\tau\langle r\rangle$ factor from the difference $1-e^{-i \tau\langle r\rangle}$, and then (6.54) follows. For the second factor, 6.54) is obtained directly.

b) We start again with 6.55), but now we can only place into $h$ the terms which have a $\tau^{2}$ factor; we call such terms negligible errors. This includes the middle term in the last expression in (6.55). Now $v$ is as in (6.48)-6.49), therefore the remaining part of the same expression can be written in the form

$$
\tau\left(l^{1} S\left(r^{-2}\right)+l^{1} S\left(r^{-1}\right) \nabla\right) v(0) e^{-i \tau\langle r\rangle}:=\tau g_{1} e^{-i \tau\langle r\rangle}, \quad g_{1} \in Z^{k, 1}
$$

and use the argument in part (a). Since $g_{1}$ already has a factor of $\tau$, the error in part (a) will have a factor of $\tau^{2}$. We further note that, compared to part (a), the error $\tau g_{1} e^{-i \tau\langle r\rangle}$ is better because it already contains the oscillatory factor $e^{-i \tau\langle r\rangle}$; we call such terms type 1 negligible errors.

It remains to consider the second term in 6.55). This is where we need to take advantage of the more precise representation for $v(0)$ in (6.48)-6.49). The contribution of $\mathrm{cr}^{-1}$ vanishes, while the contribution of $v_{2}$ in (6.48) is type 1 negligible.

Consider now the error term $\tau\left(\partial_{r}+\frac{1}{r}\right)\left(d_{i}(r) \partial_{i} r^{-1}\right) e^{-i \tau\langle r\rangle}$, arising from the second term in 6.48). We compensate for it with the explicit correction

$$
\tau v_{2}=\tau d_{i}(r) \partial_{i}(\ln r) e^{-i \tau\langle r\rangle}
$$

A direct computation shows that

$$
\begin{aligned}
P_{\tau} v_{2}-\left(\partial_{r}+\frac{1}{r}\right)\left(d_{i}(r) \partial_{i} r^{-1}\right) e^{-i \tau\langle r\rangle}= & \left(P_{\tau}-\left(\Delta+\tau^{2}\right)\right) v_{2} \\
& +\partial_{r} d_{i}\left(\partial_{i} r^{-1}\right) e^{-i \tau\langle r\rangle}+\partial_{r}^{2} d_{i} \partial_{i}(\ln r) e^{-i \tau\langle r\rangle}
\end{aligned}
$$

It suffices to show that the right hand side can be expressed in the form $g_{2} e^{-i \tau\langle r\rangle}+\tau h$ with $g_{2} \in l^{1} S\left(r^{-3}\right)$ and $h$ as in (6.54). This follows by taking into account the effect of the short and long range terms in $P_{\tau}$ for the first term, and by using the bound on $S_{r} d_{i}$ in (6.49) for the last two terms.

Finally we consider the term $\tau\left(\partial_{r}+\frac{1}{r}\right) e(r) e^{-i\langle r\rangle \tau} \in S^{-3} e^{-i\langle r\rangle \tau}$. This is the term which arises due to the long range part of $P_{\tau}$, and which is in effect responsible for the decay rate in our main theorem. Here there is no mechanism that can eliminate logarithmic type corrections for the resolvent. The redeeming feature of this case is that we can work fully with spherical symmetry, and that we can replace $P_{\tau}$ by $\Delta+\tau^{2}$ modulo negligible perturbations.

We begin by solving the equation which corresponds to $\tau=0$, namely

$$
\Delta v_{3}=\left(\partial_{r}+\frac{1}{r}\right) e(r) \in S\left(r^{-3}\right)
$$


and we obtain a solution of the form $v_{3}=\langle r\rangle^{-1} e_{1}(r)$ with $e_{1} \in S_{\text {rad }}(\log r)$. However, unlike all previous cases, here the function $\tau v_{3} e^{-i \tau\langle r\rangle}$ can no longer be a suitable correction, because that would inherit the $\log r$ term even when $\tau>r^{-1}$. Instead we consider the modified function $\tilde{v}$

$$
\tilde{v}(r, \tau)=\langle r\rangle^{-1} e_{1}\left(r \wedge|\tau|^{-1}\right)
$$

and use $v_{3}=\tau \tilde{v}(\tau, r) e^{-i \tau\langle r\rangle}$ as the correction. A direct computation shows that

$$
P_{\tau} v_{3}=\tau\left(\partial_{r}+\frac{1}{r}\right) e(r) e^{-i \tau\langle r\rangle}+2 \tau^{2}\left(\partial_{r}+\frac{1}{r}\right) \tilde{v} e^{-i \tau\langle r\rangle}+\tau g_{3}(r) e^{-i \tau\langle r\rangle}+\tau^{2} h
$$

Here $g_{3} \in l^{1} S\left(r^{-3}\right)$ and $h$ as in 6.54) are negligible errors generated by the long range and short range part of $P_{\tau}$. Thus only the second term provides non-negligible errors and needs further attention. Modulo terms which can be included in $h$, the expression $\left(\partial_{r}+\frac{1}{r}\right) \tilde{v}$ has the form

$$
\left(\partial_{r}+\frac{1}{r}\right) \tilde{v}=\chi_{<|\tau|^{-1}}(r)\langle r\rangle \partial_{r} e_{1}(r)+h
$$

Even though this comes with a $\tau^{2}$ factor, it is not quite negligible because it only has decay $\langle r\rangle^{-1} \partial_{r} e_{1} \in S\left(r^{-2}\right)$ at infinity so it logarithmically fails to be in $\mathcal{L E}^{*}$ uniformly with respect to $\tau$. Instead we need to reiterate again.

We solve

$$
\Delta e_{2}=\langle r\rangle^{-1} \partial_{r} e_{1}
$$

and obtain a solution $e_{2} \in S(\log r)$. Then we choose the last correction of the form

$$
v_{4}=\tau^{2}\langle r\rangle^{-1}\left(e_{2}\left(r \wedge|\tau|^{-1}\right)-e_{2}\left(|\tau|^{-1}\right)\right) e^{-i \tau\langle r\rangle}
$$

Here it makes no difference whether we add the factor $e^{-i \tau r}$ or not, as $v_{4}$ is supported in $\{r|\tau| \lesssim 1\}$ and does not see any oscillations. Again, a direct computation shows that

$$
P_{\tau} v_{4}=\tau^{2} \chi_{<|\tau|^{-1}}(r)\langle r\rangle \partial_{r}^{-1} e_{1} e^{-i \tau\langle r\rangle}+\tau^{2} h
$$

with $h$ as in (6.54), a negligible error. Now the first term has a better $\tau^{2}$ factor, so it is also negligible. The proof of the proposition is concluded.

\section{FROM $L^{2}$ TO POINTWISE BOUNDS}

In this section we supplement the weighted $L^{2}$ resolvent bounds with corresponding pointwise bounds. Given a $\tau$ dependent function $g(\tau)$ which is defined in $\Im \tau \leq 0$ or in an open subset of it, we look for pointwise bounds for $R_{\tau} g$ and its derivatives. First we consider the case when $g$ has only $\mathcal{L E}^{*}$ type decay at spatial infinity.

Proposition 7.16. Let $g \in \mathcal{L E}^{*}$, depending on $\tau$, so that (5.30) holds.

(i) Large $\tau,|\tau| \geq 1$. Then the following pointwise bounds are valid:

$$
\left|T^{i} \Omega^{j} S^{k} R_{\tau} g\right| \lesssim\langle r\rangle^{-1}, \quad i+4 j+16 k \leq N-12
$$


(ii) Small $\tau,|\tau| \leq 1$. Then the following pointwise bounds are valid,

$$
\left|T^{i} \Omega^{j} S^{k} R_{\tau} g\right| \lesssim\left\{\begin{array}{ll}
\min \left\{1,(|\tau|\langle r\rangle)^{-1}\right\} & i=0 \\
\langle r\rangle^{-1} & i=1 \\
\langle r\rangle^{-2}+|\tau|\langle r\rangle^{-1} & i \geq 2
\end{array} \quad i+4 j+16 k \leq N-12\right.
$$

(iii) In addition, if $\tau$ is real then the outgoing radiation condition holds:

$$
\lim _{|x| \rightarrow \infty} r\left(\partial_{r}+i \tau\right) T^{i} \Omega^{j} S^{k} R_{\tau} g=0 \quad i+4 j+16 k \leq N-12
$$

Proof. (i) Denote $v=R_{\tau} g$ and $g_{i j k}=T^{i} \Omega^{j} S^{k} R_{\tau} g, v_{i j k}=T^{i} \Omega^{j} S^{k} R_{\tau} g$. By Proposition 5.10 $g_{i j k}$ satisfy (5.31). Using the Sobolev embeddings on the sphere

$$
\|\phi\|_{L^{\infty}\left(\mathbb{S}^{2}\right)} \lesssim\|\phi\|_{L^{2}\left(\mathbb{S}^{2}\right)}+\left\|\Omega^{2} \phi\right\|_{L^{2}\left(\mathbb{S}^{2}\right)}
$$

we obtain for $i+4 j+16 k<N-12$ :

$$
\sum_{m} 2^{\frac{3 m}{2}}\left\|g_{i j k}\right\|_{L_{r}^{2} L_{\omega}^{\infty}\left(A_{m}\right)} \lesssim 1, \quad i+4 j+16 k<N-8
$$

respectively

$$
\left\|v_{i j k}\right\|_{L_{r}^{2} L_{\omega}^{\infty}\left(A_{m}\right)} \lesssim 2^{-\frac{m}{2}}, \quad i+4 j+16 k<N-12
$$

Using these bounds we revisit the equation for $v_{i j k}$ which we can rewrite in the form

$$
\left(\partial_{r}^{2}+\tau^{2}\right)\left(r v_{i j k}\right)=r^{-1} \Delta_{\omega} v_{i j k}+r Q_{l r} v_{\leq i, \leq j, \leq k}+r Q_{s r} v_{\leq i, \leq j, \leq k}+r g_{\leq i, \leq j, \leq k}
$$

In view of (7.59) and (7.60) we obtain

$$
\sum_{m} 2^{\frac{m}{2}}\left\|\left(\partial_{r}^{2}+\tau^{2}\right)\left(r v_{i j k}\right)\right\|_{L_{r}^{2} L_{\omega}^{\infty}\left(A_{m}\right)} \lesssim 1, \quad i+4 j+16 k<N-20
$$

Using (7.60) for $v_{i j k}$ and $\partial_{r} v_{i j k}$ we can localize $v_{i j k}$ so that the above bound is preserved,

$$
\left\|\left(\partial_{r}^{2}+\tau^{2}\right)\left(\chi_{A_{m}} r v_{i j k}\right)\right\|_{L_{r}^{2} L_{\omega}^{\infty}\left(A_{m}\right)} \lesssim 2^{-\frac{m}{2}}
$$

Now we use the following fundamental solution for $\partial_{r}^{2}-\tau^{2}$,

$$
K_{\tau}(s)=\tau^{-1} e^{i \tau|s|}
$$

This satisfies the bounds

$$
\left|K_{\tau}(s)\right| \lesssim|\tau|^{-1}, \quad\left|\partial_{s} K_{\tau}(s)\right| \lesssim 1
$$

Then the last bound leads to

$$
|\tau|\left\|\chi_{A_{m}} r v_{i j k}\right\|_{L^{\infty}\left(A_{j}\right)}+\left\|\partial_{r}\left(\chi_{A_{m}} r v_{i j k}\right)\right\|_{L^{\infty}\left(A_{j}\right)} \lesssim 1
$$

which we rewrite in the form

$$
|\tau|\left|v_{i j k}\right|+\left|\partial_{r} v_{i j k}\right| \lesssim\langle r\rangle^{-1}
$$

This concludes the proof of (7.56).

(ii) The arguments above remain valid in dyadic regions $A_{m}$ with $2^{m}>|\tau|^{-1}$, and directly yield (7.57) for $i=0$ and $i=1$; in the latter case we need the additional observation that $T$ can be expressed as a bounded linear combination of $\partial_{r}$ and $r^{-1} \Omega$.

Similarly, we can express $T^{2}$ in terms of $\partial_{r}^{2}, r^{-1} \partial_{r} \Omega$ and $r^{-2} \Omega^{2}$. Thus, in view of (17.57) for $i=0,1$, for the $i=2$ case of (17.57) it suffices to bound $\partial_{r}^{2} \Omega^{j} S^{k} v$. For this 
we return to (7.61), move the term $\tau^{2} v_{i j k}$ on the right and use (7.57) for $i=0,1$. This allows us to obtain a favourable bound for all terms on the right except for the $\partial_{r}^{2}$ part of $Q_{s r}$. We obtain

$$
\left\|\partial_{r}^{2} v_{0 j k}\right\|_{L^{\infty}\left(A_{m}\right)} \lesssim|\tau|\langle r\rangle^{-1}+2^{-m}\left\|\partial_{r}^{2} v_{0, \leq j, \leq k}\right\|_{L^{\infty}\left(A_{m}\right)}
$$

The second term on the right does not appear when $j=0$ and $k=0$. Hence we can use induction with respect to $j, k$ to obtain

$$
\left\|\partial_{r}^{2} v_{0 j k}\right\|_{L^{\infty}\left(A_{m}\right)} \lesssim|\tau|\langle r\rangle^{-1}
$$

and conclude the proof of (7.57) when $i=2$. The case $i>2$ is identical.

It remains to consider the case when $m$ is small, namely $2^{m} \lesssim|\tau|^{-1}$. There the argument is slightly different. Again by the Sobolev embeddings on the sphere, from (5.31) we obtain

$$
2^{-m}\left\|v_{i j k}\right\|_{L_{r}^{2} L_{\omega}^{\infty}\left(A_{m}\right)}+\left\|\nabla v_{i j k}\right\|_{L_{r}^{2} L_{\omega}^{\infty}\left(A_{m}\right)}+2^{m}\left\|\nabla^{2} v_{i j k}\right\|_{L_{r}^{2} L_{\omega}^{\infty}\left(A_{m}\right)} \lesssim 2^{-\frac{m}{2}}
$$

Then the bound (7.57) for $i=0,1$ follows by Sobolev embeddings with respect to the radial variable. Finally we obtain (17.57) for $i \geq 2$ directly from the equation (7.61) as above.

iii) Suppose $\tau$ is real. From the spherical Sobolev embeddings and the $L^{2}$ radiation condition (5.34) applied to the functions $v_{i j k}$ we obtain

$$
\lim _{m \rightarrow \infty} 2^{\frac{m}{2}}\left\|\left(\partial_{r}+i \tau\right) v_{i j k}\right\|_{L_{r}^{2} L_{\omega}^{\infty}}=0
$$

Writing $\partial_{r}^{2}+\tau^{2}=\left(\partial_{r}-i \tau\right)\left(\partial_{r}+i \tau\right)$ we interpret (7.62) as a first order ode for the functions $\left(\partial_{r}+i \tau\right)\left(r v_{i j k}\right)$. The averaged bound (7.65) allows us to integrate this ode from infinity to obtain

which implies (7.58).

$$
\lim _{r \rightarrow \infty}\left(\partial_{r}+i \tau\right)\left(r v_{i j k}\right)=0
$$

Next we consider a more favourable case when $g$ either has better decay at spatial infinity, or it has an oscillatory behavior which matches the one of the resolvent. For simplicity we restrict ourselves to real $\tau$. If $\tau$ is large then only the former alternative needs to be considered. In this case we will prove the following:

Proposition 7.17. Let $g \in Z^{m, n}$ with $m \gg n$. Then for real $\tau$ with $|\tau| \gtrsim 1$, the function $v=R_{\tau} g$ satisfies the following pointwise bounds:

$$
\left|\left(\tau \partial_{\tau}\right)^{l}\left(v e^{i \tau\langle r\rangle}\right)\right| \lesssim|\tau|^{l}\langle r\rangle^{-l-1}, \quad l \leq n
$$

Proof. We first observe that by Proposition 7.16, the bounds (7.56) hold with $N=m$. Also, by Sobolev embeddings, $g$ satisfies the stronger pointwise bounds

$$
\left|T^{i} g\right| \lesssim\langle r\rangle^{-2-i}, \quad i \leq n-2
$$

For $x$ in a compact set the exponential $e^{i \tau\langle r\rangle}$ is harmless, and on the other hand we can write $\tau \partial_{\tau}$ as a smooth combination of $S$ and $\tau T$. Hence the bound (17.66) follows from (7.56). It remains to prove (7.66) for large $r$. 
Applying (7.56) for $i=0, j=0, k=0$ we directly obtain (7.66) for $l=0$. For $l=1$ we compute

$$
\tau \partial_{\tau}\left(v e^{i \tau r}\right)=S v e^{i \tau r}+r\left(\partial_{r}+i \tau\right) v e^{i \tau r}
$$

The first term is estimated again by (7.56). Hence we need to prove that for large $r$ we have

$$
\left|\left(\partial_{r}+i \tau\right) v\right| \lesssim \frac{|\tau|}{r^{2}}
$$

For $r u$ we have in polar coordinates the equation

$$
\left(\partial_{r}^{2}+\tau^{2}\right)(r v)=r g-r^{-1} \Delta_{\omega} v+r Q_{l r} v+r Q_{s r} v
$$

Using (7.56) and (7.67) we can bound the RHS by $|\tau| r^{-2}$. Then

$$
\left|\left(\partial_{r}-i \tau\right)\left(\partial_{r}+i \tau\right)(r v)\right| \lesssim|\tau| r^{-2}
$$

which is integrated from infinity using the radiation condition to obtain (7.68). The same argument applied to the equation (7.61) for $v_{i j k}$ instead of $v$ yields

$$
\left|\left(\partial_{r}+i \tau\right) v_{i j k}\right| \lesssim \frac{|\tau|}{r^{2}}, \quad i+4 j+16 k \leq m-20
$$

For $l=2$ we compute

$$
\left(\tau \partial_{\tau}\right)^{2}\left(v e^{i \tau r}\right)=S^{2} v e^{i \tau r}+r\left(\partial_{r}+i \tau\right) S v e^{i \tau r}+r^{2}\left(\partial_{r}+i \tau\right)^{2} v e^{i \tau r}
$$

The second term is handled via (7.69) so it remains to prove that

$$
\left|\left(\partial_{r}+i \tau\right)^{2} v\right| \lesssim \frac{\tau^{2}}{r^{3}}
$$

We have

$$
\begin{aligned}
\left(\partial_{r}-i \tau\right)\left(\partial_{r}+i \tau\right)^{2}(r v)= & \left(\partial_{r}+i \tau\right)\left(r g+r^{-1} \Delta_{\omega} v+r^{-2} v+r Q_{s r} v\right) \\
= & \left(g+r^{-2} \Delta_{\omega} v+r^{-3} v+Q_{s r} u\right)+r\left(\partial_{r}+i \tau\right) f \\
& +r^{-1} \Delta_{\omega}\left(\partial_{r}+i \tau\right) v+r^{-2}\left(\partial_{r}+i \tau\right) v+r Q_{s r}\left(\partial_{r}+i \tau\right) v
\end{aligned}
$$

Since $\left(\partial_{r}+i \tau\right) v$ also satisfies the radiation condition (7.58), it suffices to show that the RHS admits a $\tau^{2} r^{-3}$ bound. This follows from the pointwise bounds (7.56) for $u$ for the terms on the first line, and (7.69) for the terms on the second line.

A similar computation using (7.61) also shows that for large $r$ we have

$$
\left|\left(\partial_{r}+i \tau\right)^{2} v_{i j k}\right| \lesssim \frac{\tau^{2}}{r^{3}}, \quad i+4 j+16 k \leq m-40
$$

The proof of (7.66) is completed by induction with respect to $l$.

Next we consider the case of small $\tau$. Here instead of the stronger decay assumption at spatial infinity we consider a weaker condition for $g$ and establish two types of regularity bounds with respect to the spectral parameter $\tau$ :

Proposition 7.18. Let $m$ be a large integer. Let $g$ be a $Z^{m, 0}$ valued function of $\tau$ in $[-1,1]$ which satisfies the additional bounds:

$$
\left\|\langle r\rangle^{n}\left(\partial_{r}+i \tau\right)^{n} T^{i} \Omega^{j} S^{k} g\right\|_{\mathcal{L} \mathcal{E}^{*}} \lesssim 1, \quad n+i+j+k \leq m
$$

Then the function $v=R_{\tau} g$ has the following properties: 
(i) $r \lesssim|\tau|^{-1}$. Then we have

$$
\left|\left(\tau \partial_{\tau}\right)^{l} v\right| \lesssim 1, \quad l \ll m
$$

(ii) $r \gtrsim|\tau|^{-1}$. Then we have

$$
\left|\left(\tau \partial_{\tau}\right)^{l}\left(v e^{i \tau\langle r\rangle}\right)\right| \lesssim(|\tau|\langle r\rangle)^{-1}, \quad l \ll m
$$

Proof. To prove the proposition we rely on the estimate (7.57) applied to the function $v$. For $g$ on the other hand, by Sobolev embeddings we have the pointwise bound

$$
\left|T^{i} \Omega^{j} S^{k}\left(g e^{i \tau\langle r\rangle}\right)\right| \lesssim\langle r\rangle^{-2-i}, \quad i+j+k \leq m-2
$$

(i). For $l=0$ the bound (7.73) follows directly from (7.57). Consider now the case $l=1$. For small $r$ we write

$$
S_{\tau} v=-S v+S_{r} v
$$

which is again bounded by (7.57). Even better, (7.57) shows that for the functions $v_{i j k}$ we have

$$
\left|S_{\tau} v_{i j k}\right|+\left|S_{r} v_{i j k}\right| \lesssim 1, \quad i+4 j+16 k \leq m-20 .
$$

For $l=2$ we write

$$
S_{\tau}^{2} v_{i j k}=S^{2} v_{i j k}-2 S_{r} S v_{i j k}+S_{r}^{2} v
$$

The first two terms are bounded as before by (7.57) and (7.76). For the third we use the equation (7.61) for $v_{i j k}$, which gives

$$
\left|S_{r}^{2} v_{i j k}\right| \lesssim r^{2} \tau^{2}\left|v_{i j k}\right|+\left|S_{r} v_{i j k}\right|+\left|\Omega^{2} v_{i j k}\right|+r^{-1} \sum_{a+b \leq 2}\left|S_{r}^{a} \Omega^{b} v_{\leq i, \leq j, \leq k}\right|+r^{2}\left|g_{\leq i, \leq j, \leq k}\right|
$$

We bound all the terms on the right via (17.57) and (17.76) to obtain

$$
\left|S_{r}^{2} v_{i j k}\right| \lesssim 1, \quad i+4 j+16 k \leq m-40 .
$$

Now we repeat the $l=2$ argument to prove by induction that

$$
\left|S_{r}^{l} v_{i j k}\right| \lesssim 1, \quad i+4 j+16 k \leq m-20 l .
$$

We remark that at each application of the equation $P_{\tau} v=g$, one or two $S_{r}$ factors are replaced by either an $\Omega$ factor or by $r \tau$ or simply by 1 .

(ii) Again the case $m=0$ follows directly from (7.57). Consider now the case $l \geq 1$. For large $r$ we compute

$$
\tau \partial_{\tau}\left(v e^{i \tau r}\right)=S v e^{i \tau r}+r\left(\partial_{r}-i \tau\right) v e^{i \tau r}
$$

Hence if $l=1$ then we need to show that

$$
\left|\left(\partial_{r}+i \tau\right) v\right| \lesssim|\tau|^{-1} r^{-2}, \quad r>|\tau|^{-1}
$$

Repeating the computation at the second and higher levels, the conclusion follows if we prove the more general bound

$$
\left|\left(\partial_{r}+i \tau\right)^{l} v_{i j k}\right| \lesssim|\tau|^{-1} r^{-1-l}, \quad r>|\tau|^{-1}, \quad i+4 j+16 k+20 l \leq m
$$

For $l=1$ we rewrite the equation for $v$ in the form

$$
\left(\partial_{r}-i \tau\right)\left(\partial_{r}+i \tau\right)(r v)=r^{-1} \Delta_{\omega} v+r Q_{l r} v+r Q_{s r} v+r g
$$


By the outgoing radiation condition (7.58) we can view this as an ode for $\left(\partial_{r}+i \tau\right)(r v)$ and integrate from infinity. By (7.57) we can bound the first three RHS terms by $|\tau|^{-1} r^{-2}$ and integrate to obtain $(|\tau| r)^{-1}$ as desired. For the $g$ term a further integration by parts is needed. Precisely, we have

$$
\int_{r_{0}}^{\infty} r g(r, \omega) e^{-i \tau r} d r=-\frac{i}{2} \tau^{-1} r_{0} g\left(r_{0}, \omega\right) e^{-i \tau r_{0}}+\int_{r_{0}}^{\infty} \frac{i}{2} \tau^{-1} r e^{-2 i \tau r} \partial_{r}\left(g(r, \omega) e^{i r \tau}\right) d r
$$

Hence by (7.75) we obtain the $(|\tau| r)^{-1}$ bound, and(7.80) is proved. We further observe that we can apply the same argument to $v_{i j k}$ using the equation (7.61) to obtain

$$
\left|\left(\partial_{r}+i \tau\right) v_{i j k}\right| \lesssim r^{-2}, \quad r>|\tau|^{-1}
$$

For $l=2$ we apply another $\partial_{r}-i \tau$ operator in (7.82). This yields

$$
\begin{aligned}
\left(\partial_{r}^{2}+\tau^{2}\right)\left(\partial_{r}+i \tau\right)(r v)= & r^{-2} \Delta_{\omega}\left(\partial_{r}+i \tau\right) v+r Q_{l r}\left(\partial_{r}+i \tau\right) v+r Q_{s r}\left(\partial_{r}+i \tau\right) v \\
& +Q_{l r} v+Q_{s r} v+\left(\partial_{r}+i \tau\right)(r g)
\end{aligned}
$$

In view of (7.57) and (7.83) we can bound all terms on the right by $|\tau|^{-1} r^{-3}$ except for the last one, and obtain (17.81) for $l=2$ integrating from infinity. For the last term, an additional integration by parts is needed, exactly as above. Applying the same argument to $v_{i j k}$ we also obtain (7.81) for $l=2$. The bound for higher $l$ is proved in an inductive manner.

\section{Conclusion}

By Lemma 3.7, the time Fourier transform $\hat{u}$ of the solution $u$ to the Cauchy problem (1.1) is represented as

$$
\hat{u}(\tau)=R_{\tau}\left(\tau u_{0}+2 P_{1} u_{0}+u_{1}\right):=R_{\tau}(\tau f+g), \quad f \in Z^{m+1,1}, \quad g \in Z^{m, 2} .
$$

The bound (1.12) will be obtained from pointwise bounds on $\hat{u}(\tau)$ and its derivatives with respect to $\tau$ by reverting the Fourier transform. This requires slightly different arguments for small and for large $\tau$.

8.1. The case of large $\tau,|\tau| \gtrsim 1$. If $\tau$ is large, then a good low frequency approximation for $\hat{u}$ is $\hat{u} \approx \tau^{-1} f$. Subtracting that, we have

$$
\hat{u}(\tau)=\tau^{-1} f+u_{1}
$$

where

$$
P_{\tau} u_{1}=\left(P^{1} f+g\right)+\tau^{-1} P^{2} f:=f_{1}+\tau^{-1} g_{1}
$$

with $f_{1} \in H^{m, 2}$ and $f_{2} \in H^{m-1,3}$. Reiterating we obtain the representation

$$
\hat{u}=\sum_{k=0}^{K-1} \tau^{-k-1} f_{k}+\tau^{-K} R_{\tau}\left(\tau f_{K}+g_{K}\right)
$$

where $f_{0}=f, f_{k} \in H^{m+1-k, k+1}$, and $g_{k} \in H^{m+1-k, k+2}$. Here we choose $K$ large but with $K \ll m$. This leads to a decomposition of the high time frequencies $P_{>1}\left(\left|D_{t}\right|\right) u$ in $u$ of the form

$$
P_{>1}\left(\left|D_{t}\right|\right) u=u_{a}+u_{b}, \quad \hat{u}_{a}=\chi>1(|\tau|) \sum_{k=0}^{K-1} \tau^{-k-1} f_{k}, \quad \hat{u}_{b}=\chi_{>1}(|\tau|) \tau^{-K} R_{\tau}\left(\tau f_{K}+g_{K}\right) .
$$


For the summands we have $\left|f_{k}\right| \lesssim\langle r\rangle^{-3-k}$ therefore applying an inverse Fourier transform we obtain

$$
\left|u_{a}\right| \lesssim\langle t\rangle^{-N}\langle r\rangle^{-3}
$$

For the resolvent expression in $u_{b}$ we use Proposition 7.17. We obtain

$$
\left|\left(\tau \partial_{\tau}\right)^{l}\left(\hat{u}_{b}(\tau) e^{i \tau r}\right)\right| \lesssim|\tau|^{-K+l}\langle r\rangle^{-1-l} .
$$

Then, inverting again the Fourier transform, we obtain the following bound:

$$
\left|u_{b}\right| \lesssim\langle r\rangle^{-1}\langle t-r\rangle^{1-K}
$$

Together, (8.86) and (8.87) give the high frequency part of (1.12).

To bound the high frequency part of $\partial_{t} u$ the same argument applies with one exception, namely the first term $f \chi_{>1}(\tau)$ arising in the expression for $\widehat{\partial_{t} u_{a}}$. However, such a term is natural since $u$ is extended by 0 to negative times therefore $\partial_{t} u$ contains a $f \delta_{t=0}$ component. Factoring that out we obtain as desired

$$
\left|P_{>1}\left(\left|D_{t}\right|\right)\left(\partial_{t} u_{a}-f \delta_{t=0}\right)\right| \lesssim\langle t\rangle^{-N}\langle r\rangle^{-4},
$$

i.e. the high frequency part of (1.13).

8.2. The case of small $\tau,|\tau| \ll 1$. Here we will still need the arguments from the large $\tau$ case, but there is an additional layer of the proof, given by the resolvent expansion around $\tau=0$ provided by Proposition 6.15. Since $f \in Z^{m+1,1}$ and $g \in Z^{m, 2}$, by applying Proposition 6.15 (a) for $f$ and Proposition 6.15 (b) for $g$ we obtain the representation

$$
\hat{u}(\tau)=\left(v+\tau v_{1}+\tau e(r, \tau)\right) e^{i \tau r}+R_{\tau}\left(\chi_{>|\tau|^{-1}}(\tau f+g)\right)+\tau^{2} R_{\tau} h
$$

with $h$ as in (6.54). Correspondingly we decompose

$$
P_{<1}\left(\left|D_{t}\right|\right) u=u_{a}+u_{b}+u_{c}+u_{d}
$$

where

$$
\begin{gathered}
\hat{u}_{a}(\tau)=\chi_{<1}(|\tau|) R_{\tau}\left(\chi_{>|\tau|^{-1}}(\tau f+g)\right), \quad \hat{u}_{b}(\tau)=\tau^{2} \chi_{<1}(|\tau|) R_{\tau} h \\
\left.\hat{u}_{c}(\tau)=\chi_{<1}(|\tau|)\left(v+\tau v_{1}\right) e^{i \tau r}, \quad \hat{u}_{d}(\tau)=\tau \chi<1(|\tau|) e(r, \tau)\right) e^{i \tau r}
\end{gathered}
$$

For the first term $u_{a}$ we use an expansion as in (8.85) to write

$$
\hat{u}_{a}=\chi_{<1}(|\tau|) \sum_{k=0}^{K-1} \tau^{-k-1} \chi_{>|\tau|^{-1}} f_{k}+\tau^{-K} \chi_{<1}(|\tau|) R_{\tau}\left(\chi_{>|\tau|^{-1}}\left(\tau f_{K}+g_{K}\right)\right)
$$

Since $f_{k} \in H^{m+1-k, k+1}$ and $g_{k} \in H^{m+1-k, k+2}$, the second term above can be included in $\hat{u}_{b}$. We remark that the spatial cutoff $\chi_{>|\tau|^{-1}}$ plays an essential role, as it allows the spatial decay of $f_{K}$ and $g_{K}$ to compensate for the $\tau^{-K}$ factor. Hence we redenote

$$
\hat{u}_{a}=\chi_{<1}(|\tau|) \sum_{k=0}^{K-1} \tau^{-k-1} \chi_{>|\tau|^{-1}} f_{k}
$$

Using the pointwise bounds $\left|f_{k}\right| \lesssim\langle r\rangle^{-3-k}$ we invert the Fourier transform to obtain as in the large $\tau$ case

$$
\left|u_{a}\right| \lesssim\langle r\rangle^{-3}\left(\frac{\langle r\rangle}{\langle r\rangle+t}\right)^{N}, \quad\left|\partial_{t} u_{a}-P_{<1}\left(\left|D_{t}\right|\right) f \delta_{t=0}\right| \lesssim\langle r\rangle^{-3}\left(\frac{\langle r\rangle}{\langle r\rangle+t}\right)^{N}
$$


For $u_{b}$ we use Proposition 7.18 to obtain

$$
\left|\left(\tau \partial_{\tau}\right)^{l} \hat{u}_{b}\right| \lesssim \tau^{2}, \quad|\tau|<\langle r\rangle^{-1}
$$

and

$$
\left|\left(\tau \partial_{\tau}\right)^{l}\left(\hat{u}_{b} e^{i r \tau}\right)\right| \lesssim \tau r^{-1}, \quad|\tau|>\langle r\rangle^{-1}
$$

which lead to

$$
\left|u_{b}\right| \lesssim\langle r\rangle^{-1}\langle t-r\rangle^{-2}, \quad\left|\partial_{t} u_{b}\right| \lesssim\langle r\rangle^{-1}\langle t-r\rangle^{-3}
$$

For $u_{c}$ we have the pointwise bounds $\left|v_{0}\right| \lesssim 1,\left|v_{1}\right| \lesssim 1$ therefore we easily get

$$
\left|u_{c}\right|+\left|\partial_{t} u_{c}\right| \lesssim\langle r\rangle^{-1}\langle t-r\rangle^{-N}
$$

Finally we consider $u_{d}$, which in this analysis represents the leading term of the contribution to $u$ of the long range terms in the operator $P$.

We recall that the expression $e(r, \tau)$ has the form

$$
e(r, \tau)=\langle r\rangle^{-1} e_{1}\left(r \wedge|\tau|^{-1}\right)+\tau\left(e_{2}\left(r \wedge|\tau|^{-1}\right)-e_{2}\left(|\tau|^{-1}\right)\right), \quad e_{1}, e_{2} \in S(\log r)
$$

We represent $e_{1}$ and $e_{2}$ in the form

$$
e_{12}(r)=\sum_{k \geq 0} c_{k} \chi_{>k}(r), \quad\left|c_{k}\right| \lesssim 1,
$$

where $\chi_{>k}$ are bump functions which equal 0 for $r \ll 2^{m}$, equal 1 for $r \gg 2^{m}$ and are smooth on the $2^{m}$ scale. We denote by $e_{k}$ the contributions of each summand to $e$ and consider three cases depending on the size of $r$ :

i) $r \ll 2^{k}$. Then $e_{k}$ has the form

$$
e_{k}(r, \tau)=c_{k} \tau^{2} \chi_{<2^{-k}}(|\tau|)
$$

therefore its corresponding output in $u_{d}$ satisfies

$$
\left|u_{d}^{k}\right|+2^{k}\left|\partial_{t} u_{d}^{k}\right| \lesssim 2^{-3 k}\left(1+2^{k} t\right)^{-N}
$$

which leads to (1.12) and (1.13) after summation with respect to $k$ with $2^{k}>r$.

ii) $r \gg 2^{k}$. Then $e_{k}$ has the form

$$
e_{k}(r, \tau)=c_{k} \tau\langle r\rangle^{-1} \chi_{<2^{-k}}(|\tau|)
$$

therefore its corresponding output in $u_{d}$ satisfies

$$
\left|u_{d}^{k}\right|+2^{k}\left|\partial_{t} u_{d}^{k}\right| \lesssim\langle r\rangle^{-1} 2^{-2 k}\left(1+2^{k} t\right)^{-N}
$$

which leads again to (1.12) and (1.13) since now we are summing with respect to $k$ for $2^{k}<r$.

iii) $r \approx 2^{k}$. Here we get terms as in both (i) and (ii), but there is no summation with respect to $k$.

The proof of Theorem 1.4 is concluded. 


\section{REFERENCES}

[1] Serge Alinhac. On the Morawetz-Keel-Smith-Sogge inequality for the wave equation on a curved background. Publ. Res. Inst. Math. Sci., 42(3):705-720, 2006.

[2] Lars Andersson and Pieter Blue. Hidden symmetries and decay for the wave equation on the Kerr spacetime. arXiv:0908.2265.

[3] P. Blue and A. Soffer. Semilinear wave equations on the Schwarzschild manifold. I. Local decay estimates. Adv. Differential Equations, 8(5):595-614, 2003.

[4] P. Blue and A. Soffer. The wave equation on the Schwarzschild metric. II. Local decay for the spin-2 Regge-Wheeler equation. J. Math. Phys., 46(1):012502, 9, 2005.

[5] Pieter Blue and Jacob Sterbenz. Uniform decay of local energy and the semi-linear wave equation on Schwarzschild space. Comm. Math. Phys., 268(2):481-504, 2006.

[6] Nicolas Burq, Fabrice Planchon, John G. Stalker, and A. Shadi Tahvildar-Zadeh. Strichartz estimates for the wave and Schrödinger equations with potentials of critical decay. Indiana Univ. Math. J., 53(6):1665-1680, 2004.

[7] E. S. C. Ching, P. T. Leung, W. M. Suen, and K. Young. Late-time tail of wave propagation on curved spacetime. Phys. Rev. Lett., 74(13):2414-2417, Mar 1995.

[8] E. S. C. Ching, P. T. Leung, W. M. Suen, and K. Young. Quasinormal mode expansion for linearized waves in gravitational systems. Phys. Rev. Lett., 74(23):4588-4591, Jun 1995.

[9] Hans Christianson. Dispersive estimates for manifolds with one trapped orbit. Comm. Partial Differential Equations, 33(7-9):1147-1174, 2008.

[10] Demetrios Christodoulou and Sergiu Klainerman. The global nonlinear stability of the Minkowski space, volume 41 of Princeton Mathematical Series. Princeton University Press, Princeton, NJ, 1993.

[11] Yves Colin de Verdière and Bernard Parisse. Équilibre instable en régime semi-classique. I. Concentration microlocale. Comm. Partial Differential Equations, 19(9-10):1535-1563, 1994.

[12] Mihalis Dafermos and Igor Rodnianski. Lectures on black holes and linear waves. arXiv:0811.0354.

[13] Mihalis Dafermos and Igor Rodnianski. A note on energy currents and decay for the wave equation on a Schwarzschild background. arXiv:0710.0171v1.

[14] Mihalis Dafermos and Igor Rodnianski. A proof of the uniform boundedness of solutions to the wave equation on slowly rotating Kerr backgrounds. arXiv:0805.4309.

[15] Mihalis Dafermos and Igor Rodnianski. A proof of Price's law for the collapse of a self-gravitating scalar field. Invent. Math., 162(2):381-457, 2005.

[16] Mihalis Dafermos and Igor Rodnianski. The red-shift effect and radiation decay on black hole spacetimes. Comm. Pure Appl. Math., 62(7):859-919, 2009.

[17] F. Finster, N. Kamran, J. Smoller, and S.-T. Yau. Decay of solutions of the wave equation in the Kerr geometry. Comm. Math. Phys., 264(2):465-503, 2006.

[18] Felix Finster and Joel Smoller. A time-independent energy estimate for outgoing scalar waves in the Kerr geometry. J. Hyperbolic Differ. Equ., 5(1):221-255, 2008.

[19] S. W. Hawking and G. F. R. Ellis. The large scale structure of space-time. Cambridge University Press, London, 1973. Cambridge Monographs on Mathematical Physics, No. 1.

[20] Fritz John. Blow-up of solutions of nonlinear wave equations in three space dimensions. Manuscripta Math., 28(1-3):235-268, 1979.

[21] Bernard S. Kay and Robert M. Wald. Linear stability of Schwarzschild under perturbations which are nonvanishing on the bifurcation 2-sphere. Classical Quantum Gravity, 4(4):893-898, 1987.

[22] Markus Keel, Hart F. Smith, and Christopher D. Sogge. On global existence for nonlinear wave equations outside of convex obstacles. Amer. J. Math., 122(4):805-842, 2000.

[23] Markus Keel, Hart F. Smith, and Christopher D. Sogge. Almost global existence for some semilinear wave equations. J. Anal. Math., 87:265-279, 2002. Dedicated to the memory of Thomas H. Wolff.

[24] S. Klainerman. Remarks on the global Sobolev inequalities in the Minkowski space $\mathbf{R}^{n+1}$. Comm. Pure Appl. Math., 40(1):111-117, 1987.

[25] Johann Kronthaler. Decay rates for spherical scalar waves in the Schwarzschild geometry. preprint, arXiv:0709.3703.

[26] Johann Kronthaler. The Cauchy problem for the wave equation in the Schwarzschild geometry. $J$. Math. Phys., 47(4):042501, 29, 2006.

[27] I. Laba and A. Soffer. Global existence and scattering for the nonlinear Schrödinger equation on Schwarzschild manifolds. Helv. Phys. Acta, 72(4):274-294, 1999. 
[28] Jonathan Luk. Improved decay for solutions to the linear wave equation on a schwarzschild black hole. arXiv:0906.5588.

[29] Jeremy Marzuola, Jason Metcalfe, and Daniel Tataru. Strichartz estimates and local smoothing estimates for asympototically flat Schrödinger equations. J. Funct. Anal., 255(6):1497-1553, 2008.

[30] Jeremy Marzuola, Jason Metcalfe, Daniel Tataru, and Mihai Tohaneanu. Strichartz estimates on Schwarzschild black hole backgrounds. Comm. Math. Phys., 293(1):37-83, 2010.

[31] Jason Metcalfe and Christopher D. Sogge. Long-time existence of quasilinear wave equations exterior to star-shaped obstacles via energy methods. SIAM J. Math. Anal., 38(1):188-209 (electronic), 2006.

[32] Jason Metcalfe and Christopher D. Sogge. Global existence of null-form wave equations in exterior domains. Math. Z., 256(3):521-549, 2007.

[33] Jason Metcalfe and Daniel Tataru. Decay estimates for variable coefficient wave equations in exterior domains. to appear, Progress in Nonlinear Differential Equations and Their Applications; arXiv:0806.3409, 2008.

[34] Jason Metcalfe and Daniel Tataru. Global parametrices and dispersive estimates for variable coefficient wave equations. to appear, Math. Ann., arXiv:0707.1191, 2007.

[35] Cathleen S. Morawetz. Exponential decay of solutions of the wave equation. Comm. Pure Appl. Math., 19:439-444, 1966.

[36] Cathleen S. Morawetz. Time decay for the nonlinear Klein-Gordon equations. Proc. Roy. Soc. Ser. A, 306:291-296, 1968.

[37] Cathleen S. Morawetz. Decay for solutions of the exterior problem for the wave equation. Comm. Pure Appl. Math., 28:229-264, 1975.

[38] A. Soffer P. Blue. Improved decay rates with small regularity loss for the wave equation about a Schwarzschild black hole. preprint, http://xxx.lanl.gov, math/0612168, 2006.

[39] Richard H. Price. Nonspherical perturbations of relativistic gravitational collapse. I. Scalar and gravitational perturbations. Phys. Rev. D (3), 5:2419-2438, 1972.

[40] Avy Soffer Roland Donninger, Wilhelm Schlag. A proof of price's law on Schwarzschild black hole manifolds for all angular momenta. arXiv:0908.4292.

[41] W. Schlag. Dispersive estimates for Schrödinger operators: a survey. In Mathematical aspects of nonlinear dispersive equations, volume 163 of Ann. of Math. Stud., pages 255-285. Princeton Univ. Press, Princeton, NJ, 2007.

[42] Maciej Zworski Stephane Nonnenmacher. Semiclassical resolvent estimates in chaotic scattering. arXiv:0904.2986.

[43] Walter A. Strauss. Dispersal of waves vanishing on the boundary of an exterior domain. Comm. Pure Appl. Math., 28:265-278, 1975.

[44] Walter A. Strauss and Kimitoshi Tsutaya. Existence and blow up of small amplitude nonlinear waves with a negative potential. Discrete Contin. Dynam. Systems, 3(2):175-188, 1997.

[45] N. Szpak. Weighted- $l^{\infty}$ and pointwise space-time decay estimates for wave equations with potentials and initial data of low regularity. preprint (2007). arXiv: math-ph/0708.2801.

[46] Nikodem Szpak. Linear and nonlinear tails. I. General results and perturbation theory. J. Hyperbolic Differ. Equ., 5(4):741-765, 2008

[47] Nikodem Szpak, Piotr Bizoń, Tadeusz Chmaj, and Andrzej Rostworowski. Linear and nonlinear tails. II. Exact decay rates and spherical symmetry. J. Hyperbolic Differ. Equ., 6(1):107-125, 2009.

[48] Daniel Tataru. Parametrices and dispersive estimates for Schrödinger operators with variable coefficients. Amer. J. Math., 130(3):571-634, 2008.

[49] Daniel Tataru and Mihai Tohaneanu. Local energy estimates on Kerr black hole backgrounds. IMRN, to appear; arXiv:0810.5766.

[50] Mihai Tohaneanu. Local energy estimates and Strichartz estimates on Schwarzschild and Kerr black hole backgrounds. Thesis, University of California Berkeley 2009.

[51] Mihai Tohaneanu. Strichartz estimates on Kerr black hole backgrounds. arXiv:0910.1545, 2009.

[52] F. Twainy. The time decay of solutions to the scalar wave equation in Schwarzschild background. Thesis, University of California San Diego 1989.

[53] Robert M. Wald. Note on the stability of the Schwarzschild metric. J. Math. Phys., 20(6):1056-1058, 1979 .

Department of Mathematics, University of California, Berkeley, CA 94720-3840 